March 2003

\title{
Reflection-Transmission Algebras
}

\author{
M. Mintchev ${ }^{a 1}$, E. Ragoucy ${ }^{b 2}$ and P. Sorba ${ }^{c 3}$ \\ a INFN and Dipartimento di Fisica, Universitá di Pisa, Via Buonarroti 2, \\ 56127 Pisa, Italy \\ ${ }^{b}$ LAPTH, 9, Chemin de Bellevue, BP 110, F-74941 Annecy-le-Vieux cedex, \\ France \\ c TH Division, CERN, CH 1211 Geneva 23, Switzerland
}

\begin{abstract}
Inspired by factorized scattering from delta-type impurities in $(1+1)$-dimensional space-time, we propose and analyse a generalization of the Zamolodchikov-Faddeev algebra. Distinguished elements of the new algebra, called reflection and transmission generators, encode the particle-impurity interactions. We describe in detail the underlying algebraic structure. The relative Fock representations are explicitly constructed and a general factorized scattering theory is developed in this framework.
\end{abstract}

CERN-TH/2003-058

IFUP-TH 14/2003

LAPTH-972/03

hep-th/0303187

\footnotetext{
${ }^{1}$ mintchev@df.unipi.it

${ }^{2}$ ragoucy@lapp.in2p3.fr

${ }^{3}$ sorba@cern.ch and sorba@lapp.in2p3.fr, on leave of absence from LAPTH
} 


\section{Introduction}

Much progress has been made in the last two decades in understanding the physical properties and the mathematical structure of integrable quantum systems in $1+1$ dimensions. The idea of factorized scattering, which can be traced back to the pioneering work of Yang [1], plays a central rôle in most of the significant developments in this field. It has been recognized later, that the algebraic structure in the basis of factorized scattering theory is the Zamolodchikov-Faddeev (ZF) algebra [2]-[4]. This algebra represents a powerful tool for deriving not only $S$-matrix amplitudes, but also formfactors of local operators [5, 6].

Integrable models with boundaries [7]-[14] or defects [15]-[21] have recently also been the subject of intense study. Since factorized scattering turns out to be fundamental in this context as well, the natural problem that arises is to find the counterpart of the ZF algebra, which works in the presence of reflecting and transmitting impurities. The main goal of the present paper is to introduce such an algebra, called in what follows reflection-transmission (RT) algebra. Our strategy is to generalize the approach to integrable systems on the half-line developed in [14. Besides the particle creation and annihilation operators, the RT algebra involves also reflection and transmission (defect) generators. In the Fock representation, the latter acquire non-vanishing vacuum expectation values, defined in terms of the observable reflection and transmission amplitudes of a single particle interacting with the defect. Together with the two-body bulk scattering matrix, these amplitudes form the physical input. The structure of the RT algebra is inspired by some exactly solvable integrable models with $\delta$-type impurities. Apart from providing a useful test for the general setup, these systems find concrete applications [17, 20] in conductance problems.

The paper is organized as follows. In the next section we focus on quantum inverse scattering with $\delta$-impurities. The general concept of RT algebra is introduced in section 3. After a brief description of the basic features of these algebras, we construct the relative Fock representations. Section 4 is devoted to scattering with impurities. We first establish the unitarity and factorization constraints. Using the Fock representation of a suitable RT algebra, we define afterwards the "in"-coming and "out"-going states and construct the total scattering operator. The last section contains our conclusions. We discuss there some universal features of the RT algebras and their relevance to inverse scattering and other related topics. 


\section{Origin of reflection-transmission algebras}

It is instructive to start the discussion with two examples, showing how the RT algebras emerge from the study of quantum impurity problems. We begin with the $n$-particle Hamiltonian

$$
H^{(n)}=\sum_{i=1}^{n}-\frac{1}{2} \partial_{x_{i}}^{2}+\eta \delta\left(x_{i}\right), \quad \eta \in \mathbb{R}
$$

which describes a system of $n$ non-relativistic bosonic particles (of unit mass) on $\mathbb{R}$, which interact with a $\delta$-type impurity localized in the origin, but not among themselves. This model is well-known to be exactly solvable. It is sufficient to investigate the spectral problem associated with the one-particle Hamiltonian $H^{(1)}$, defined on a suitable (see e.g. [22]) domain $\mathcal{D}_{\eta} \subset L^{2}(\mathbb{R}, d x)$ of continuous functions on $\mathbb{R}$, which are twice differentiable in $\mathbb{R} \backslash\{0\}$ and satisfy

$$
\lim _{x \downarrow 0}\left[\left(\partial_{x} \psi\right)(x)-\left(\partial_{x} \psi\right)(-x)\right]=2 \eta \psi(0) .
$$

$H^{(1)}$ is self-adjoint on $\mathcal{D}_{\eta}$. A set of orthogonal (generalized) eigenstates $\left\{\psi_{k}^{ \pm}(x): k \in \mathbb{R}\right\}$, verifying (2.2), is

$$
\psi_{k}^{ \pm}(x)=\theta(\mp k)\left\{\theta(\mp x) T(\mp k) \mathrm{e}^{i k x}+\theta( \pm x)\left[\mathrm{e}^{i k x}+R(\mp k) \mathrm{e}^{-i k x}\right]\right\},
$$

where $\theta$ denotes the standard Heaviside function and

$$
T(k)=\frac{k}{k+i \eta}, \quad R(k)=\frac{-i \eta}{k+i \eta} .
$$

The family $\left\{\bar{\psi}_{-k}^{ \pm}(x): k \in \mathbb{R}\right\}$, where the bar stands for complex conjugation, is also orthonormal. The systems $\left\{\psi_{k}^{ \pm}(x): k \in \mathbb{R}\right\}$ and $\left\{\bar{\psi}_{-k}^{ \pm}(x): k \in \mathbb{R}\right\}$ represent physically scattering states and, for $\eta \geq 0$, are separately complete in $L^{2}(\mathbb{R}, d x)$. When $\eta<0$, there is in addition one bound state

$$
\psi_{\mathrm{b}}(x)=\theta(-\eta) \sqrt{|\eta|}\left[\theta(x) \mathrm{e}^{\eta x}+\theta(-x) \mathrm{e}^{-\eta x}\right]
$$

which is orthogonal to $\left\{\psi_{k}^{ \pm}(x): k \in \mathbb{R}\right\}$ and $\left\{\bar{\psi}_{-k}^{ \pm}(x): k \in \mathbb{R}\right\}$. The energy of $\psi_{\mathrm{b}}$ is $E=-\eta^{2} / 2$.

Particle collision and production processes are absent from this simple model. Nevertheless, the reflection and transmission from the impurity give 
rise to a non-trivial scattering operator, which preserves the particle number and can be described as follows. Using the weak limits

$$
\lim _{x \rightarrow \pm \infty} \frac{\mathrm{e}^{ \pm i k x}}{k+i \epsilon}=0, \quad \epsilon>0
$$

one can verify that

$$
\lim _{x \rightarrow \pm \infty}\left[\psi_{k}^{ \pm}(x)-\mathrm{e}^{i k x}\right]=0 .
$$

Therefore, one can interpret $\psi_{k}^{ \pm}(x)$ asymptotically as incoming waves, travelling in $\mathbb{R}_{ \pm}$with momentum $k \neq 0$ towards the impurity. Accordingly, we take the vectors

$$
|k\rangle^{\text {in }}=\psi_{k}^{+}(x)+\psi_{k}^{-}(x),
$$

to be the basis of one-particle "in" states. Analogous considerations lead us to choose the following basis of one-particle "out" states:

$$
|k\rangle^{\text {out }}=\bar{\psi}_{-k}^{+}(x)+\bar{\psi}_{-k}^{-}(x) .
$$

The one-particle scattering operator is defined at this point by

$$
\mathbf{S}^{(1)}|k\rangle^{\text {out }}=|k\rangle^{\text {in }} .
$$

By construction, $\mathbf{S}^{(1)}$ is a unitary operator on $L^{2}(\mathbb{R}, d x)$ for $\eta \geq 0$. In the range $\eta<0, \mathbf{S}^{(1)}$ is defined and unitary on the orthogonal complement to the bound state (2.5) in $L^{2}(\mathbb{R}, d x)$. The one-particle transition amplitude reads

$$
\begin{gathered}
{ }^{\text {out }}\langle p \mid k\rangle^{\text {in }}={ }^{\text {out }}\left\langle p\left|\mathbf{S}^{(1)}\right| k\right\rangle^{\text {out }}= \\
{[\theta(p) T(p)+\theta(-p) T(-p)] 2 \pi \delta(p-k)+} \\
{[\theta(p) R(p)+\theta(-p) R(-p)] 2 \pi \delta(p+k),}
\end{gathered}
$$

which clarifies the physical meaning of $T$ and $R$, given by eq. (2.4). They represent the transmission and reflection amplitudes and admit a meromorphic continuation in $k$ to the whole complex plane $\mathbb{C}$. The pole $k=-i \eta$ confirms the presence of the bound state $\psi_{\mathrm{b}}$ for $\eta<0$ and indicates the existence of a resonance state for $\eta>0$.

The $n$-particle amplitude, with initial and final configurations satisfying $k_{1}<\ldots<k_{n}$ and $p_{1}>\ldots>p_{n}$ respectively, can be expressed in terms of (2.11) as follows:

$$
{ }^{\text {out }}\left\langle p_{1}, \ldots, p_{n} \mid k_{1}, \ldots, k_{n}\right\rangle^{\text {in }}={ }^{\text {out }}\left\langle p_{1}, \ldots, p_{n}\left|\mathbf{S}^{(n)}\right| k_{1}, \ldots, k_{n}\right\rangle^{\text {out }}=\prod_{i=1}^{n}{ }^{\text {out }}\left\langle p_{i} \mid k_{i}\right\rangle^{\text {in }} \text {. }
$$


Equation (2.12) concludes our brief summary of the standard and well-known analytic treatment of the integrable system defined by (2.1).

A natural question one may ask at this point concerns the existence of an algebraic framework for dealing with the above system for $\eta \neq 0$, similar to the familiar canonical commutation approach, which works in the case $\eta=0$. The answer to this question turns out to be affirmative and we now turn to the description of the relevant algebraic structure. Following our previous work 21, we introduce the associative algebra $\mathcal{C}_{B}$ with identity element $\mathbf{1}$, generated by $\left\{a^{* \xi}(k), a_{\xi}(k): \xi= \pm, k \in \mathbb{R}\right\}$ obeying the bosonic-type commutation relations:

$$
\begin{gathered}
a_{\xi_{1}}\left(k_{1}\right) a_{\xi_{2}}\left(k_{2}\right)-a_{\xi_{2}}\left(k_{2}\right) a_{\xi_{1}}\left(k_{1}\right)=0, \\
a^{* \xi_{1}}\left(k_{1}\right) a^{* \xi_{2}}\left(k_{2}\right)-a^{* \xi_{2}}\left(k_{2}\right) a^{* \xi_{1}}\left(k_{1}\right)=0, \\
a_{\xi_{1}}\left(k_{1}\right) a^{* \xi_{2}}\left(k_{2}\right)-a^{* \xi_{2}}\left(k_{2}\right) a_{\xi_{1}}\left(k_{1}\right)= \\
{\left[\delta_{\xi_{1}}^{\xi_{2}}+\mathcal{T}_{\xi_{1}}^{\xi_{2}}\left(k_{1}\right)\right] 2 \pi \delta\left(k_{1}-k_{2}\right) \mathbf{1}+\mathcal{R}_{\xi_{1}}^{\xi_{2}}\left(k_{1}\right) 2 \pi \delta\left(k_{1}+k_{2}\right) \mathbf{1},}
\end{gathered}
$$

where

$$
\mathcal{T}(k)=\left(\begin{array}{cc}
0 & T(k) \\
\bar{T}(k) & 0
\end{array}\right), \quad \mathcal{R}(k)=\left(\begin{array}{cc}
R(k) & 0 \\
0 & \bar{R}(k)
\end{array}\right) .
$$

The right-hand side of eq. (2.15) captures the presence of the impurity. The term proportional to $\delta\left(k_{1}+k_{2}\right)$ reflects in particular the breaking of translation and Galilean invariance due to the impurity. We shall see in the next section that $\mathcal{C}_{B}$ is a particular RT algebra. For the moment we focus on the Fock representation $\mathcal{F}_{\mathcal{R}, \mathcal{T}}\left(\mathcal{C}_{\mathcal{B}}\right)$ of $\mathcal{C}_{B}$, referring for the explicit construction to sect. 3.2. An essential feature of $\mathcal{F}_{\mathcal{R}, \mathcal{T}}\left(\mathcal{C}_{\mathcal{B}}\right)$ is that the operators $\left\{a^{* \xi}(k), a_{\xi}(k)\right\}$ in this representation satisfy

$$
\begin{aligned}
a_{\xi}(k) & =\mathcal{T}_{\xi}^{\eta}(k) a_{\eta}(k)+\mathcal{R}_{\xi}^{\eta}(k) a_{\eta}(-k), \\
a^{* \xi}(k) & =a^{* \eta}(k) \mathcal{T}_{\eta}^{\xi}(k)+a^{* \eta}(-k) \mathcal{R}_{\eta}^{\xi}(-k) .
\end{aligned}
$$

Hereafter the summation over repeated upper and lower indices is understood. The relations (2.17), (2.18) originate from the reflection-transmission automorphism characterizing any RT algebra and established in sect. 3. In the physical context these relations encode the interaction with the impurity.

The vacuum state $\Omega \in \mathcal{F}_{\mathcal{R}, \mathcal{T}}\left(\mathcal{C}_{\mathcal{B}}\right)$ obeys as usual $a_{\xi}(k) \Omega=0$. We denote by $(\cdot, \cdot)$ the scalar product in $\mathcal{F}_{\mathcal{R}, \mathcal{T}}\left(\mathcal{C}_{\mathcal{B}}\right)$ and consider the vacuum expectation value

$$
\left(a^{* \eta_{1}}\left(p_{1}\right) \ldots a^{* \eta_{n}}\left(p_{n}\right) \Omega, a^{* \xi_{1}}\left(k_{1}\right) \ldots a^{* \xi_{n}}\left(k_{n}\right) \Omega\right),
$$


with

$$
k_{1}<\cdots<k_{n}, \quad \xi_{i}=-\epsilon\left(k_{i}\right), \quad p_{1}>\cdots>p_{n}, \quad \eta_{i}=\epsilon\left(p_{i}\right),
$$

$\epsilon$ being the sign function. By means of eqs. (2.13)-(2.15) it is easily verified that (2.19) precisely reproduce the amplitudes (2.12) for any $n$. Therefore, $\mathcal{C}_{B}$ provides a purely algebraic framework for constructing the scattering operator. The formalism actually applies to any observable of the system, introducing in addition to $\mathcal{C}_{B}$ the creation and annihilation operators $\left\{b^{*}, b\right\}$ for the bound state (2.5), which commute with $\left\{a^{* \xi}(k), a_{\xi}(k)\right\}$ and satisfy

$$
[b, b]=\left[b^{*}, b^{*}\right]=0, \quad\left[b, b^{*}\right]=1 .
$$

For the Hamiltonian one finds, for instance,

$$
H=\frac{1}{2} \int_{-\infty}^{+\infty} \frac{d k}{2 \pi} k^{2} a^{* \xi}(k) a_{\xi}(k)-\theta(-\eta) \frac{\eta^{2}}{2} b^{*} b .
$$

The restriction of $H$ to the $n$-particle subspace of the total Hilbert space (including the bound state) is the algebraic counterpart of the Hamiltonian (2.1) we started with.

At this stage we have enough background to turn to quantum field theory with $\delta$-type impurities [15, 20]. Our goal will be to demonstrate that the algebra $\mathcal{C}_{B}$ can be successfully applied there as well. As an example we consider the model

$$
\begin{gathered}
S[\varphi]=\frac{1}{2} \int_{-\infty}^{+\infty} d t \int_{-\infty}^{+\infty} d x\left[\left(\partial_{t} \varphi\right)^{2}(t, x)-\left(\partial_{x} \varphi\right)^{2}(t, x)\right. \\
\left.-m^{2} \varphi^{2}(t, x)-2 \eta \delta(x) \varphi^{2}(t, x)\right],
\end{gathered}
$$

with $m \geq 0$ and $\eta \in \mathbb{R}$. The action (2.23) defines a standard external field problem with $\delta$-potential. The corresponding equation of motion is

$$
\left[\partial_{t}^{2}-\partial_{x}^{2}+m^{2}+2 \eta \delta(x)\right] \varphi(t, x)=0
$$

and our problem will now be to quantize (2.24) with the standard initial conditions:

$$
\left[\varphi\left(0, x_{1}\right), \varphi\left(0, x_{2}\right)\right]=0, \quad\left[\left(\partial_{t} \varphi\right)\left(0, x_{1}\right), \varphi\left(0, x_{2}\right)\right]=-i \delta\left(x_{1}-x_{2}\right) .
$$

The solution of this problem requires the study of the operator

$$
K \equiv-\partial_{x}^{2}+m^{2}+2 \eta \delta(x)
$$


We already know that $K$ is self-adjoint on $\mathcal{D}_{\eta}$. In order to avoid imaginary energies, we demand $K$ to be non-negative, which implies

$$
-m \leq \eta \text {. }
$$

Now, the solution of eqs. (2.24) and (2.25) is unique and can be expressed in terms of the generators $\left\{a^{* \xi}(k), a_{\xi}(k)\right\}$ and $\left\{b^{*}, b\right\}$. One finds

$$
\varphi(t, x)=\varphi_{+}(t, x)+\varphi_{-}(t, x)+\varphi_{\mathrm{b}}(t, x),
$$

where

$$
\begin{gathered}
\varphi_{ \pm}(t, x)=\int_{-\infty}^{+\infty} \frac{d k}{2 \pi \sqrt{2 \omega(k)}}\left[a^{* \pm}(k) \bar{\psi}_{k}^{ \pm}(x) \mathrm{e}^{i \omega(k) t}+a_{ \pm}(k) \psi_{k}^{ \pm}(x) \mathrm{e}^{-i \omega(k) t}\right] \\
\varphi_{\mathrm{b}}(t, x)=\frac{1}{\sqrt{2 \omega(i \eta)}}\left[b^{*} \mathrm{e}^{i t \omega(i \eta)}+b \mathrm{e}^{-i t \omega(i \eta)}\right] \psi_{\mathrm{b}}(x),
\end{gathered}
$$

with $\omega(k)=\sqrt{k^{2}+m^{2}}$.

Using eqs. (2.15) and (2.28)-(2.30), one easily derives the two-point vacuum expectation value

$$
\begin{aligned}
& w^{(2)}\left(t_{1}, x_{1}, t_{2}, x_{n}\right)=\left(\varphi\left(t_{1}, x_{1}\right) \Omega, \varphi\left(t_{2}, x_{2}\right) \Omega\right)= \\
& \int_{-\infty}^{+\infty} \frac{d k}{4 \pi \omega(k)} \mathrm{e}^{-i \omega(k) t_{12}}\left\{\theta\left(x_{1}\right) \theta\left(-x_{2}\right) T(k) \mathrm{e}^{i k x_{12}}+\theta\left(-x_{1}\right) \theta\left(x_{2}\right) \bar{T}(k) \mathrm{e}^{i k x_{12}}+\right. \\
& \left.\theta\left(x_{1}\right) \theta\left(x_{2}\right)\left[\mathrm{e}^{i k x_{12}}+R(k) \mathrm{e}^{i k \widetilde{x}_{12}}\right]+\theta\left(-x_{1}\right) \theta\left(-x_{2}\right)\left[\mathrm{e}^{i k x_{12}}+\bar{R}(k) \mathrm{e}^{i k \widetilde{x}_{12}}\right]\right\}+ \\
& \frac{1}{2 \omega(i \eta)} \mathrm{e}^{-i t_{12} \omega(i \eta)} \psi_{\mathrm{b}}\left(x_{1}\right) \psi_{\mathrm{b}}\left(x_{2}\right),
\end{aligned}
$$

where $t_{12}=t_{1}-t_{2}, x_{12}=x_{1}-x_{2}$ and $\widetilde{x}_{12}=x_{1}+x_{2}$. The last term in (2.31) represents the contribution of the bound state and vanishes for $\eta \geq 0$. The field $\varphi$ has a relativistic dispersion relation $\omega(k)^{2}=k^{2}+m^{2}$, but nevertheless Lorentz invariance is manifestly broken in (2.31).

The function (2.31) fully determines the theory. In fact $w^{(2 n+1)}=0$, whereas $w^{(2 n)}$ can be derived from $w^{(2)}$ by means of the well-known recursion relation

$$
\begin{gathered}
w^{(2 n)}\left(t_{1}, x_{1}, \ldots, t_{2 n}, x_{2 n}\right)= \\
\sum_{i=1}^{2 n-1} w^{(2)}\left(t_{i}, x_{i}, t_{2 n}, x_{2 n}\right) w^{(2 n-2)}\left(t_{1}, x_{1}, \ldots, \widehat{t}_{i}, \widehat{x}_{i}, \ldots, t_{2 n-1}, x_{2 n-1}\right),
\end{gathered}
$$


the hat indicating that the corresponding argument must be omitted.

Having at our disposal all correlation functions, we can derive the scattering operator of the theory. The particles of our model do not interact directly, but interact with the external $\delta$-function field, modelling the impurity. We will now show that the associated scattering matrix is fully determined by the algebra $\mathcal{C}_{B}$. Equation (2.29) therefore represents a true quantum inverse scattering transform, allowing a reconstruction of the fields $\varphi_{ \pm}$from $\left\{a^{* \xi}(k), a_{\xi}(k)\right\}$. Let us concentrate first on the case $\eta \geq 0$, commenting at the end on the range $-m \leq \eta<0$. In developing the scattering theory one can use the Haag-Ruelle approach [23] with some minor modifications 24, which reflect the absence of translation invariance. The novel feature, with respect to the quantum mechanical example discussed above, is that in quantum field theory we need smearing with special wave-packets for the free Klein-Gordon equation, which keep trace of the position $x=0$ of the impurity. Such wave-packets can be introduced as follows. Let $\mathcal{D}(\mathbb{R})$ be the space of smooth test functions with compact support. Then

$$
f^{t}(x)=\int_{-\infty}^{+\infty} \frac{d k}{2 \pi \sqrt{2 \omega(k)}} f(k) \mathrm{e}^{i k x-i \omega(k) t}, \quad f \in \mathcal{D}(\mathbb{R}),
$$

is a smooth solution of the Klein-Gordon equation of mass $m$. We will say that $f_{1} \in \mathcal{D}(\mathbb{R})$ precedes $f_{2} \in \mathcal{D}(\mathbb{R})$ and write $f_{1} \prec f_{2}$ if and only if $\operatorname{supp}\left(f_{1}\right) \cap \operatorname{supp}\left(f_{2}\right)=\emptyset$ and $k_{1}<k_{2}$ for all $k_{1} \in \operatorname{supp}\left(f_{1}\right)$ and all $k_{2} \in \operatorname{supp}\left(f_{2}\right)$. We now introduce the two sets $\left\{g_{i}(k) \in \mathcal{D}(\mathbb{R}): i=1, \ldots, m\right\}$ and $\left\{h_{j}(k) \in \mathcal{D}(\mathbb{R}): j=1, \ldots, n\right\}$, which satisfy the non-overlapping conditions

$$
\begin{array}{rlrl}
g_{1} \prec \cdots \prec g_{m}, & h_{n} & \prec \cdots \prec h_{1}, \\
0 & \notin \operatorname{supp} g_{i}, & 0 & \notin \operatorname{supp} h_{j} .
\end{array}
$$

Setting now

$$
\xi_{i}=\left\{\begin{array}{rl}
+, & \operatorname{supp} g_{i} \subset \mathbb{R}_{-}, \\
-, & \operatorname{supp} g_{i} \subset \mathbb{R}_{+},
\end{array} \quad \eta_{j}=\left\{\begin{aligned}
+, & \operatorname{supp} h_{j} \subset \mathbb{R}_{+}, \\
-, & \operatorname{supp} h_{j} \subset \mathbb{R}_{-},
\end{aligned}\right.\right.
$$

we define

$$
g_{\xi_{i}}^{t}(x)=\theta\left(\xi_{i} x\right) g_{i}^{t}(x), \quad h_{\eta_{j}}^{t}(x)=\theta\left(\eta_{j} x\right) h_{j}^{t}(x) .
$$

By construction, $g_{\xi_{i}}^{t}(x)$ represent wave-packets in $\mathbb{R}_{\xi_{i}}$ which move towards the impurity in $x=0$. On the other hand, $h_{\eta_{j}}^{t}(x)$ are wave-packets in $\mathbb{R}_{\eta_{j}}$, 
which travel away from the impurity in the direction $x \rightarrow \eta_{j} \infty$. Therefore one expects that the smeared operators

$$
\begin{aligned}
& \varphi\left(t, g_{\xi_{i}}^{t}\right)=i \int_{-\infty}^{+\infty} d x\left[\left(\partial_{t} g_{\xi_{i}}^{t}\right)(x) \varphi(t, x)-g_{\xi_{i}}^{t}(x)\left(\partial_{t} \varphi\right)(t, x)\right], \\
& \varphi\left(t, h_{\eta_{j}}^{t}\right)=i \int_{-\infty}^{+\infty} d x\left[\left(\partial_{t} h_{\eta_{j}}^{t}\right)(x) \varphi(t, x)-h_{\eta_{j}}^{t}(x)\left(\partial_{t} \varphi\right)(t, x)\right]
\end{aligned}
$$

generate asymptotic "in" and "out" states respectively. This is indeed the case because of the existence of the following strong limits in the Fock space $\mathcal{F}_{\mathcal{R}, \mathcal{T}}\left(\mathcal{C}_{\mathcal{B}}\right)$

$$
\begin{gathered}
\lim _{t \rightarrow-\infty} \varphi\left(t, g_{\xi_{1}}^{t}\right) \cdots \varphi\left(t, g_{\xi_{m}}^{t}\right) \Omega=a^{* \xi_{1}}\left(g_{1}\right) \cdots a^{* \xi_{m}}\left(g_{m}\right) \Omega \equiv\left|g_{1}, \ldots, g_{m}\right\rangle^{\text {in }} \\
\lim _{t \rightarrow+\infty} \varphi\left(t, h_{\eta_{1}}^{t}\right) \cdots \varphi\left(t, h_{\eta_{n}}^{t}\right) \Omega=a^{* \eta_{1}}\left(h_{1}\right) \cdots a^{* \eta_{n}}\left(h_{n}\right) \Omega \equiv\left|h_{1}, \ldots, h_{n}\right\rangle^{\text {out }}
\end{gathered}
$$

where

$$
a^{* \zeta}(f)=\int_{-\infty}^{+\infty} \frac{d k}{2 \pi} f(k) a^{* \zeta}(k) .
$$

Let us sketch the proof of (2.39), for example. Using the non-overlapping conditions (2.34), the commutation relations (2.13)-(2.15) and the constraints (2.17), (2.18), one first derives the identity

$$
\varphi\left(t, g_{\xi_{1}}^{t}\right) \cdots \varphi\left(t, g_{\xi_{m}}^{t}\right) \Omega=a^{* \xi_{1}}\left(\widetilde{g}_{\xi_{1}}^{t}\right) \cdots a^{* \xi_{m}}\left(\widetilde{g}_{\xi_{m}}^{t}\right) \Omega
$$

where

$$
\widetilde{g}_{\xi_{i}}^{t}(p)=\int_{-\infty}^{\infty} d x \theta\left(\xi_{i} x\right) \int_{-\infty}^{\infty} \frac{d k}{2 \pi} \frac{\omega(k)+\omega(p)}{2 \sqrt{\omega(k) \omega(p)}} g_{i}(k) \mathrm{e}^{i[\omega(p)-\omega(k)] t-i x(p-k)} .
$$

Therefore, eq. (2.39) is equivalent to

$$
\lim _{t \rightarrow-\infty}\left\|a^{* \xi_{1}}\left(\widetilde{g}_{\xi_{1}}^{t}\right) \cdots a^{* \xi_{m}}\left(\widetilde{g}_{\xi_{m}}^{t}\right) \Omega-a^{* \xi_{1}}\left(g_{1}\right) \cdots a^{* \xi_{m}}\left(g_{m}\right) \Omega\right\|=0
$$

$\|\cdot\|$ being the $L^{2}$-norm. For proving (2.44) one uses the continuity of $a^{* \zeta}(f)$ in $f$ (see the estimate (3.61) below) and

$$
\lim _{t \rightarrow-\infty}\left\|\widetilde{g}_{\xi_{i}}^{t}-g_{i}\right\|=0, \quad \forall i=1, \ldots, m
$$


Finally, (2.45) is a consequence of the weak limits (2.6),$x$ being replaced by $t$. This concludes the argument.

Summarizing, the finite linear combinations of the vectors

$$
\begin{aligned}
& \left.\left\{\left|g_{1}, \ldots, g_{m}\right\rangle\right\rangle^{\text {in }}: g_{1} \prec \cdots \prec g_{m}, m=1,2, \ldots\right\}, \\
& \left\{\left|h_{1}, \ldots, h_{n}\right\rangle^{\text {out }}: h_{n} \prec \cdots \prec h_{1}, n=1,2, \ldots\right\}
\end{aligned}
$$

generate, after completion with respect to the scalar product in the Fock space $\mathcal{F}_{\mathcal{R}, \mathcal{T}}\left(\mathcal{C}_{\mathcal{B}}\right)$, the asymptotic spaces $\mathcal{H}^{\text {in }}$ and $\mathcal{H}^{\text {out }}$. It turns out that asymptotic completeness $\mathcal{H}^{\text {out }}=\mathcal{F}_{\mathcal{R}, \mathcal{T}}\left(\mathcal{C}_{\mathcal{B}}\right)=\mathcal{H}^{\text {in }}$ holds for $\eta \geq 0$. The transition amplitudes read

$$
{ }^{\text {out }}\left\langle h_{1}, \ldots, h_{n} \mid g_{1}, \ldots, g_{m}\right\rangle^{\text {in }}=\delta_{m n} \prod_{i=1}^{n} \int_{-\infty}^{+\infty} \frac{d p_{i}}{2 \pi} \frac{d k_{i}}{2 \pi} \bar{h}_{i}\left(p_{i}\right)^{\text {out }}\left\langle p_{i} \mid k_{i}\right\rangle^{\text {in }} g_{i}\left(k_{i}\right) \text {, }
$$

where ${ }^{\text {out }}\langle p \mid k\rangle^{\text {in }}$ is given by (2.11). These results can be generalized to the case $-m<\eta<0$, except for the property of asymptotic completeness, which is violated by the bound state present in this range.

It is worth mentioning that the above framework applies with straightforward modifications to fermionic systems as well. The relevant algebra $\mathcal{C}_{F}$ is obtained in this case simply by replacing the commutators in the right-hand sides of eqs. (2.13)-(2.15) with anticommutators.

It is evident from the above considerations that $\mathcal{C}_{B}$ is a universal and powerful tool for handling $\delta$-type impurities, both in quantum mechanics and quantum field theory. One can view $\mathcal{C}_{B}\left(\mathcal{C}_{F}\right)$ as a central extension of the algebra of canonical commutation (anticommutation) relations. A direct generalization, emerging at this point, is to substitute $\mathcal{T}(k) \mathbf{1}$ and $\mathcal{R}(k) \mathbf{1}$ in eq. (2.15) with new generators $t(k)$ and $r(k)$, which are no longer central elements. Moreover, in the spirit of the ZF algebra, it is possible to replace the bosonic (fermionic) exchange factor between $\left\{a^{* \xi}(k), a_{\xi}(k)\right\}$ with a more general one. In this way one naturally arrives at the general concept of RT algebra, described in the next section.

\section{$3 \quad$ Reflection-transmission algebras}

\subsection{Definition and general properties}

Inspired by the above treatment of $\delta$-impurities, we introduce an associative algebra with identity element 1 and two types of generators, $\left\{a_{\alpha}(\chi), a^{* \alpha}(\chi)\right\}$ 
and $\left\{r_{\alpha}^{\beta}(\chi), t_{\alpha}^{\beta}(\chi)\right\}$, called bulk and defect (reflection and transmission) generators, respectively. We refer to $\chi \in \mathbb{R}$ as a spectral parameter. In the context of inverse scattering $\chi$ parametrizes the particle dispersion relation (see eqs. (4.1) below). To be able to deal with systems with internal degrees of freedom, we adopt double indices $\alpha=(\xi, i)$. The component $\xi= \pm$ indicates the half-line $\mathbb{R}_{ \pm}$where the particle is created or annihilated, whereas $i=1, \ldots, N$ parametrizes the internal ("isotopic") degrees of freedom. The generators $\left\{a_{\alpha}(\chi), a^{* \alpha}(\chi), r_{\alpha}^{\beta}(\chi), t_{\alpha}^{\beta}(\chi)\right\}$ are subject to the following constraints:

- bulk exchange relations

$$
\begin{aligned}
& a_{\alpha_{1}}\left(\chi_{1}\right) a_{\alpha_{2}}\left(\chi_{2}\right)-\mathcal{S}_{\alpha_{2} \alpha_{1}}^{\beta_{1} \beta_{2}}\left(\chi_{2}, \chi_{1}\right) a_{\beta_{2}}\left(\chi_{2}\right) a_{\beta_{1}}\left(\chi_{1}\right)=0, \\
& a^{* \alpha_{1}}\left(\chi_{1}\right) a^{* \alpha_{2}}\left(\chi_{2}\right)-a^{* \beta_{2}}\left(\chi_{2}\right) a^{* \beta_{1}}\left(\chi_{1}\right) \mathcal{S}_{\beta_{2} \beta_{1}}^{\alpha_{1} \alpha_{2}}\left(\chi_{2}, \chi_{1}\right)=0, \\
& a_{\alpha_{1}}\left(\chi_{1}\right) a^{* \alpha_{2}}\left(\chi_{2}\right)-a^{* \beta_{2}}\left(\chi_{2}\right) \mathcal{S}_{\alpha_{1} \beta_{2}}^{\alpha_{2} \beta_{1}}\left(\chi_{1}, \chi_{2}\right) a_{\beta_{1}}\left(\chi_{1}\right)= \\
& 2 \pi \delta\left(\chi_{1}-\chi_{2}\right)\left[\delta_{\alpha_{1}}^{\alpha_{2}} \mathbf{1}+t_{\alpha_{1}}^{\beta_{2}}\left(\chi_{1}\right)\right]+2 \pi \delta\left(\chi_{1}+\chi_{2}\right) r_{\alpha_{1}}^{\alpha_{2}}\left(\chi_{1}\right) ;
\end{aligned}
$$

- defect exchange relations

$$
\begin{gathered}
\mathcal{S}_{\alpha_{1} \alpha_{2}}^{\gamma_{2} \gamma_{1}}\left(\chi_{1}, \chi_{2}\right) r_{\gamma_{1}}^{\delta_{1}}\left(\chi_{1}\right) \mathcal{S}_{\gamma_{2} \delta_{1}}^{\beta_{1} \delta_{2}}\left(\chi_{2},-\chi_{1}\right) r_{\delta_{2}}^{\beta_{2}}\left(\chi_{2}\right)= \\
r_{\alpha_{2}}^{\gamma_{2}}\left(\chi_{2}\right) \mathcal{S}_{\alpha_{1} \gamma_{2}}^{\delta_{2} \delta_{1}}\left(\chi_{1},-\chi_{2}\right) r_{\delta_{1}}^{\gamma_{1}}\left(\chi_{1}\right) \mathcal{S}_{\delta_{2} \gamma_{1}}^{\beta_{1} \beta_{2}}\left(-\chi_{2},-\chi_{1}\right) ; \\
\mathcal{S}_{\alpha_{1} \alpha_{2}}^{\gamma_{2} \gamma_{1}}\left(\chi_{1}, \chi_{2}\right) t_{\gamma_{1}}^{\delta_{1}}\left(\chi_{1}\right) \mathcal{S}_{\gamma_{2} \delta_{1}}^{\beta_{1} \delta_{2}}\left(\chi_{2}, \chi_{1}\right) t_{\delta_{2}}^{\beta_{2}}\left(\chi_{2}\right)= \\
t_{\alpha_{2}}^{\gamma_{2}}\left(\chi_{2}\right) \mathcal{S}_{\alpha_{1} \gamma_{2}}^{\delta_{2} \delta_{1}}\left(\chi_{1}, \chi_{2}\right) t_{\delta_{1}}^{\gamma_{1}}\left(\chi_{1}\right) \mathcal{S}_{\delta_{2} \gamma_{1}}^{\beta_{1} \beta_{2}}\left(\chi_{2}, \chi_{1}\right) ; \\
\mathcal{S}_{\alpha_{1} \alpha_{2}}^{\gamma_{2} \gamma_{1}}\left(\chi_{1}, \chi_{2}\right) t_{\gamma_{1}}^{\delta_{1}}\left(\chi_{1}\right) \mathcal{S}_{\gamma_{2} \delta_{1}}^{\beta_{1} \delta_{2}}\left(\chi_{2}, \chi_{1}\right) r_{\delta_{2}}^{\beta_{2}}\left(\chi_{2}\right)= \\
r_{\alpha_{2}}^{\gamma_{2}}\left(\chi_{2}\right) \mathcal{S}_{\alpha_{1} \gamma_{2}}^{\delta_{2} \delta_{1}}\left(\chi_{1},-\chi_{2}\right) t_{\delta_{1}}^{\gamma_{1}}\left(\chi_{1}\right) \mathcal{S}_{\delta_{2} \gamma_{1}}^{\beta_{1} \beta_{2}}\left(-\chi_{2}, \chi_{1}\right) ;
\end{gathered}
$$

- mixed exchange relations

$$
\begin{gathered}
a_{\alpha_{1}}\left(\chi_{1}\right) r_{\alpha_{2}}^{\beta_{2}}\left(\chi_{2}\right)=\mathcal{S}_{\alpha_{2} \alpha_{1}}^{\gamma_{1} \gamma_{2}}\left(\chi_{2}, \chi_{1}\right) r_{\gamma_{2}}^{\delta_{2}}\left(\chi_{2}\right) \mathcal{S}_{\gamma_{1} \delta_{2}}^{\beta_{2} \delta_{1}}\left(\chi_{1},-\chi_{2}\right) a_{\delta_{1}}\left(\chi_{1}\right) \\
r_{\alpha_{1}}^{\beta_{1}}\left(\chi_{1}\right) a^{* \alpha_{2}}\left(\chi_{2}\right)=a^{* \delta_{2}}\left(\chi_{2}\right) \mathcal{S}_{\alpha_{1} \delta_{2}}^{\gamma_{2} \delta_{1}}\left(\chi_{1}, \chi_{2}\right) r_{\delta_{1}}^{\gamma_{1}}\left(\chi_{1}\right) \mathcal{S}_{\gamma_{2} \gamma_{1}}^{\beta_{1} \alpha_{2}}\left(\chi_{2},-\chi_{1}\right) \\
a_{\alpha_{1}}\left(\chi_{1}\right) t_{\alpha_{2}}^{\beta_{2}}\left(\chi_{2}\right)=\mathcal{S}_{\alpha_{2} \alpha_{1}}^{\gamma_{1} \gamma_{2}}\left(\chi_{2}, \chi_{1}\right) t_{\gamma_{2}}^{\delta_{2}}\left(\chi_{2}\right) \mathcal{S}_{\gamma_{1} \delta_{2}}^{\beta_{2} \delta_{1}}\left(\chi_{1}, \chi_{2}\right) a_{\delta_{1}}\left(\chi_{1}\right) \\
t_{\alpha_{1}}^{\beta_{1}}\left(\chi_{1}\right) a^{* \alpha_{2}}\left(\chi_{2}\right)=a^{* \delta_{2}}\left(\chi_{2}\right) \mathcal{S}_{\alpha_{1} \delta_{2}}^{\gamma_{2} \delta_{1}}\left(\chi_{1}, \chi_{2}\right) t_{\delta_{1}}^{\gamma_{1}}\left(\chi_{1}\right) \mathcal{S}_{\gamma_{2} \gamma_{1}}^{\beta_{1} \alpha_{2}}\left(\chi_{2}, \chi_{1}\right)
\end{gathered}
$$


The exchange factor $\mathcal{S}$ is required to satisfy some compatibility conditions, which read as follows, in conventional tensor notation:

$$
\begin{gathered}
\mathcal{S}_{12}\left(\chi_{1}, \chi_{2}\right) \mathcal{S}_{12}\left(\chi_{2}, \chi_{1}\right)=\mathbb{I} \otimes \mathbb{I} \\
\mathcal{S}_{12}\left(\chi_{1}, \chi_{2}\right) \mathcal{S}_{23}\left(\chi_{1}, \chi_{3}\right) \mathcal{S}_{12}\left(\chi_{2}, \chi_{3}\right)=\mathcal{S}_{23}\left(\chi_{2}, \chi_{3}\right) \mathcal{S}_{12}\left(\chi_{1}, \chi_{3}\right) \mathcal{S}_{23}\left(\chi_{1}, \chi_{2}\right)
\end{gathered}
$$

Equation (3.11) is know as the "unitarity" condition, whereas (3.12) is the celebrated quantum Yang-Baxter equation in its braid form, $\mathbb{R}$ playing the role of spectral set. We emphasize that $\mathcal{S}$ depends in general on $\chi_{1}$ and $\chi_{2}$ separately, which allows [25] to treat systems with broken Lorentz (Galilean) invariance - an expected feature when defects are present.

Recapitulating, with any solution $\mathcal{S}$ of (3.11), (3.12) we associate an associative algebra $\mathcal{C}_{\mathcal{S}}$, whose generators $\left\{a_{\alpha}(\chi), a^{* \alpha}(\chi), r_{\alpha}^{\beta}(\chi), t_{\alpha}^{\beta}(\chi)\right\}$ satisfy the constraints (3.1)-(3.10). The bulk exchange relations (3.1)-(3.3) are similar to those of the ZF algebra, but for the presence of the defect generators in the right-hand side of (3.3). The exchange properties of the latter are described by eqs. (3.4) - (3.6). Equation (3.4) looks similar to the boundary Yang-Baxter equation [7, the difference being that in general the elements $\left\{r_{\alpha}^{\beta}(\chi)\right\}$ do not commute and, consequently, their position in (3.4) is essential. Notice that $\left\{r_{\alpha}^{\beta}(\chi), t_{\alpha}^{\beta}(\chi)\right\}$ close a subalgebra of $\mathcal{C}_{\mathcal{S}}$, which generalizes the Sklyanin algebra [9] for the pure reflection case $\left(t_{\alpha}^{\beta}(\chi)=0\right)$. The mixed relations (3.7)- (3.10) complete the list, fixing the exchange properties between bulk and defect generators.

Two particular cases of $\mathcal{C}_{\mathcal{S}}$ were previously investigated. Setting $t_{\alpha}^{\beta}(\chi)=0$ one gets the boundary algebra introduced in [14 for handling integrable systems on the half-line $\mathbb{R}_{+}$. For $r_{\alpha}^{\beta}(\chi)=t_{\alpha}^{\beta}(\chi)=0$ one obtains instead the $\mathrm{ZF}$ algebra, which applies to the same systems, but on the whole line $\mathbb{R}$. In this respect $\mathcal{C}_{\mathcal{S}}$ emerges as a unifying algebraic structure for quantum field inverse scattering in $1+1$ dimensions, which works also in the presence of impurities. This expectation is widely confirmed by the results reported in sect. 4.

In this paper we focus on RT algebras. A RT algebra is a $\mathcal{C}_{\mathcal{S}}$-algebra whose defect generators satisfy in addition

$$
\begin{gathered}
t_{\alpha_{1}}^{\beta}(\chi) t_{\beta}^{\alpha_{2}}(\chi)+r_{\alpha_{1}}^{\beta}(\chi) r_{\beta}^{\alpha_{2}}(-\chi)=\delta_{\alpha_{1}}^{\alpha_{2}}, \\
t_{\alpha_{1}}^{\beta}(\chi) r_{\beta}^{\alpha_{2}}(\chi)+r_{\alpha_{1}}^{\beta}(\chi) t_{\beta}^{\alpha_{2}}(-\chi)=0 .
\end{gathered}
$$


A characteristic feature of any RT algebra is a peculiar automorphism, which implements in algebraic terms the physical concepts of transmission and reflection and which is established a few lines below.

For constructing the Fock representation of $\mathcal{C}_{\mathcal{S}}$, one needs an involution. The most natural one is obtained by extending the mapping

$$
\begin{array}{ll}
I: a^{* \alpha}(\chi) \mapsto a_{\alpha}(\chi), & I: a_{\alpha}(\chi) \mapsto a^{* \alpha}(\chi), \\
I: r_{\alpha}^{\beta}(\chi) \mapsto r_{\beta}^{\alpha}(-\chi), & I: t_{\alpha}^{\beta}(\chi) \mapsto t_{\beta}^{\alpha}(\chi),
\end{array}
$$

as an antilinear antihomomorphism on $\mathcal{C}_{\mathcal{S}}$. In fact, it is not difficult to check that $I$ leaves (3.1)-(3.13) invariant, provided that

$$
\mathcal{S}_{12}^{\dagger}\left(\chi_{1}, \chi_{2}\right)=\mathcal{S}_{12}\left(\chi_{2}, \chi_{1}\right)
$$

where the dagger stands for the Hermitian conjugation. The condition (3.17), known 2] as Hermitian analyticity of $\mathcal{S}$, is assumed in what follows.

Let us finally consider the mapping

$$
\begin{gathered}
\varrho: a_{\alpha}(\chi) \mapsto t_{\alpha}^{\beta}(\chi) a_{\beta}(\chi)+r_{\alpha}^{\beta}(\chi) a_{\beta}(-\chi), \\
\varrho: a^{* \alpha}(\chi) \mapsto a^{* \beta}(\chi) t_{\beta}^{\alpha}(\chi)+a^{* \beta}(-\chi) r_{\beta}^{\alpha}(-\chi), \\
\varrho: r_{\alpha}^{\beta}(\chi) \mapsto r_{\alpha}^{\beta}(\chi), \quad \varrho: t_{\alpha}^{\beta}(\chi) \mapsto t_{\alpha}^{\beta}(\chi) .
\end{gathered}
$$

One can directly verify that $\varrho$ leaves (3.1)-(3.13) invariant as well therefore and extends to an automorphism on $\mathcal{C}_{\mathcal{S}}$, considered as an algebra with involution $I$. We refer to $\varrho$ as reflection-transmission automorphism and remark that because of (3.13) and (3.14), $\varrho$ is idempotent. Equations (3.18) and (3.19) are the algebraic counterparts of (2.17) and (2.18). They have a simple physical interpretation: each particle in the bulk is $\varrho$-equivalent to a superposition of a transmitted and reflected particle.

$\mathcal{C}_{\mathcal{S}}$ is an infinite algebra and from the above formal definition it is not obvious at all that it has an operator realization. Since such a realization is needed in the physical applications, we will construct in the next section an explicit representation of $\mathcal{C}_{\mathcal{S}}$ in terms of (generally unbounded) operators, which act in a Hilbert space. 


\subsection{Fock representation}

We consider below representations of the RT algebra with involution $\left\{\mathcal{C}_{\mathcal{S}}, I\right\}$

with the following structure:

(i) the representation space is a complex Hilbert space $\mathcal{H}$ with scalar product $(\cdot, \cdot)$

(ii) the generators $\left\{a_{\alpha}(\chi), a^{* \alpha}(\chi), r_{\alpha}^{\beta}(\chi), t_{\alpha}^{\beta}(\chi)\right\}$ are operator-valued distributions with common and invariant dense domain $\mathcal{D} \subset \mathcal{H}$, where eqs. (3.1) - (3.14) hold;

(iii) the involution $I$ is realized as a conjugation with respect to $(\cdot, \cdot)$.

A Fock representation is further specified by the condition:

(iv) there exists a vacuum state $\Omega \in \mathcal{D}$, which is annihilated by $a_{\alpha}(\chi)$. The vector $\Omega$ is cyclic with respect to $\left\{a^{* \alpha}(\chi)\right\}$ and $(\Omega, \Omega)=1$.

There is a number of simple, but quite important consequences from the assumptions (i-iv). We start with

Proposition 3.1 The reflection-transmission automorphism @ is realized in any Fock representation by the identity operator.

Proof. We consider the matrix element

$$
\left(\varphi,\left\{a_{\alpha}\left(\chi_{1}\right)-\varrho\left[a_{\alpha}\left(\chi_{1}\right)\right]\right\} P\left[a^{*}\right] \Omega\right),
$$

where $\varphi$ is an arbitrary state in $\mathcal{D}$ and $P$ is an arbitrary polynomial in $a^{*}$. Applying the identity

$$
\left\{a_{\alpha}\left(\chi_{1}\right)-\varrho\left[a_{\alpha}\left(\chi_{1}\right)\right]\right\} a^{* \beta}\left(\chi_{2}\right)=a^{* \gamma}\left(\chi_{2}\right) \mathcal{S}_{\alpha \gamma}^{\beta \delta}\left(\chi_{1}, \chi_{2}\right)\left\{a_{\delta}\left(\chi_{1}\right)-\varrho\left[a_{\delta}\left(\chi_{1}\right)\right]\right\},
$$

which follows after some algebra from the exchange relations (3.3), (3.8), (3.10), we can shift the curly bracket in (3.21) to the vacuum and deduce from (iv) that

$$
\left(\varphi,\left\{a_{\alpha}(\chi)-\varrho\left[a_{\alpha}(\chi)\right]\right\} P\left[a^{*}\right] \Omega\right)=0 .
$$

Taking the complex conjugate of (3.23), we obtain

$$
\left(P\left[a^{*}\right] \Omega,\left\{a^{* \alpha}(\chi)-\varrho\left[a^{* \alpha}(\chi)\right]\right\} \varphi\right)=0 .
$$


Finally, using the cyclicity of $\Omega$, we conclude that

$$
a^{* \alpha}(\chi)=\varrho\left[a^{* \alpha}(\chi)\right]=a^{* \beta}(\chi) t_{\beta}^{\alpha}(\chi)+a^{* \beta}(-\chi) r_{\beta}^{\alpha}(-\chi)
$$

holds on $\mathcal{D}$. Analogously, we derive

$$
a_{\alpha}(\chi)=\varrho\left[a_{\alpha}(\chi)\right]=t_{\alpha}^{\beta}(\chi) a_{\beta}(\chi)+r_{\alpha}^{\beta}(\chi) a_{\beta}(-\chi) .
$$

Notice that the reflection-transmission identities (3.25), (3.26) generalize the $\delta$-impurity relations (2.17), (2.18).

In what follows we are going to show that any RT algebra $\mathcal{C}_{\mathcal{S}}$ admits in general a whole family $\mathcal{F}\left(\mathcal{C}_{\mathcal{S}}\right)$ of Fock representations, which can be parametrized by means of the vacuum expectation values

$$
\mathcal{R}_{\alpha}^{\beta}(\chi)=\left(\Omega, r_{\alpha}^{\beta}(\chi) \Omega\right), \quad \mathcal{T}_{\alpha}^{\beta}(\chi)=\left(\Omega, t_{\alpha}^{\beta}(\chi) \Omega\right),
$$

called in what follows transmission and reflection matrices. Their basic properties are collected in

Proposition 3.2 In each Fock representation of $\left\{\mathcal{C}_{\mathcal{S}}, I\right\}$ :

(a) $\mathcal{T}(\chi)$ and $\mathcal{R}(\chi)$ satisfy the Hermitian analyticity conditions

$$
\begin{gathered}
\mathcal{R}^{\dagger}(\chi)=\mathcal{R}(-\chi), \\
\mathcal{T}^{\dagger}(\chi)=\mathcal{T}(\chi) ;
\end{gathered}
$$

(b) the vacuum state $\Omega$ is unique (up to a phase factor) and satisfies

$$
r_{\alpha}^{\beta}(\chi) \Omega=\mathcal{R}_{\alpha}^{\beta}(\chi) \Omega, \quad t_{\alpha}^{\beta}(\chi) \Omega=\mathcal{T}_{\alpha}^{\beta}(\chi) \Omega .
$$

(c) $\mathcal{T}(\chi)$ and $\mathcal{R}(\chi)$ obey the consistency relations

$$
\begin{array}{r}
\mathcal{S}_{12}\left(\chi_{1}, \chi_{2}\right) \mathcal{R}_{2}\left(\chi_{1}\right) \mathcal{S}_{12}\left(\chi_{2},-\chi_{1}\right) \mathcal{R}_{2}\left(\chi_{2}\right)= \\
\mathcal{R}_{2}\left(\chi_{2}\right) \mathcal{S}_{12}\left(\chi_{1},-\chi_{2}\right) \mathcal{R}_{2}\left(\chi_{1}\right) \mathcal{S}_{12}\left(-\chi_{2},-\chi_{1}\right), \\
\mathcal{S}_{12}\left(\chi_{1}, \chi_{2}\right) \mathcal{T}_{2}\left(\chi_{1}\right) \mathcal{S}_{12}\left(\chi_{2}, \chi_{1}\right) \mathcal{T}_{2}\left(\chi_{2}\right)= \\
\mathcal{T}_{2}\left(\chi_{2}\right) \mathcal{S}_{12}\left(\chi_{1}, \chi_{2}\right) \mathcal{T}_{2}\left(\chi_{1}\right) \mathcal{S}_{12}\left(\chi_{2}, \chi_{1}\right),
\end{array}
$$




$$
\begin{gathered}
\mathcal{S}_{12}\left(\chi_{1}, \chi_{2}\right) \mathcal{T}_{2}\left(\chi_{1}\right) \mathcal{S}_{12}\left(\chi_{2}, \chi_{1}\right) \mathcal{R}_{2}\left(\chi_{2}\right)= \\
\mathcal{R}_{2}\left(\chi_{2}\right) \mathcal{S}_{12}\left(\chi_{1},-\chi_{2}\right) \mathcal{T}_{2}\left(\chi_{1}\right) \mathcal{S}_{12}\left(-\chi_{2}, \chi_{1}\right),
\end{gathered}
$$

and unitarity conditions

$$
\begin{aligned}
& \mathcal{T}(\chi) \mathcal{T}(\chi)+\mathcal{R}(\chi) \mathcal{R}(-\chi)=\mathbb{I} \\
& \mathcal{T}(\chi) \mathcal{R}(\chi)+\mathcal{R}(\chi) \mathcal{T}(-\chi)=0 .
\end{aligned}
$$

Proof. The statement (a) is a direct consequence of (3.15) and point (iii) above. Concerning (b), the argument implying the uniqueness of the vacuum is standard (see e.g. 26]). The identities in (3.30) can be deduced from

$$
\left(\left[r_{\alpha}^{\beta}(\chi)-\mathcal{R}_{\alpha}^{\beta}(\chi)\right] \Omega, P\left[a^{*}\right] \Omega\right)=0
$$

and

$$
\left(\left[t_{\alpha}^{\beta}(\chi)-\mathcal{T}_{\alpha}^{\beta}(\chi)\right] \Omega, P\left[a^{*}\right] \Omega\right)=0
$$

respectively, $P$ being an arbitrary polynomial. For proving (3.36) and (3.37), one can shift by Hermitian conjugation the polynomial to the first factor and use afterwards the exchange relations (3.7) and (3.9) and eq. (3.27). Finally, (b) can be verified by taking the vacuum expectation values of (3.4)-(3.6) and (3.13), (3.14) and using (3.30). This concludes the argument.

We thus recover at the level of Fock representation the well-known boundary Yang-Baxter equation (3.31). A novel feature is the presence of transmission (3.32) and transmission-reflection (3.33) Yang-Baxter equations. Using (3.11), eq. (3.33) can be equivalently rewritten in the form

$$
\begin{array}{r}
\mathcal{S}_{12}\left(\chi_{1}, \chi_{2}\right) \mathcal{R}_{2}\left(\chi_{1}\right) \mathcal{S}_{12}\left(\chi_{2},-\chi_{1}\right) \mathcal{T}_{2}\left(\chi_{2}\right)= \\
\mathcal{T}_{2}\left(\chi_{2}\right) \mathcal{S}_{12}\left(\chi_{1}, \chi_{2}\right) \mathcal{R}_{2}\left(\chi_{1}\right) \mathcal{S}_{12}\left(\chi_{2},-\chi_{1}\right) .
\end{array}
$$

Let us elaborate now a bit more on the relation between $\mathcal{R}$ and $\mathcal{T}$. Because of (3.28), (3.29), $\mathcal{T}(\chi) \mathcal{T}(\chi)$ and $\mathcal{R}(\chi) \mathcal{R}(-\chi)$ are non-negative Hermitian matrices which, according to (3.34), are simultaneously diagonalizable. The corresponding eigenvalues satisfy

$$
\lambda_{i}(\chi)+\mu_{i}(\chi)=1, \quad \lambda_{i}(\chi) \geq 0, \quad \mu_{i}(\chi) \geq 0, \quad i=1, \ldots, N .
$$


Solving eq. (3.34) for $\mathcal{T}$, one finds

$$
\mathcal{T}(\chi)=\tau(\chi) \sqrt{\mathbb{I}-\mathcal{R}(\chi) \mathcal{R}(-\chi)}=\tau(\chi) \sum_{n=0}^{\infty} \alpha_{n}[\mathcal{R}(\chi) \mathcal{R}(-\chi)]^{n},
$$

where $\tau(\chi)$ is some unknown function and the coefficients $\alpha_{n}$ are determined by $\sqrt{1-x}=\sum_{n=0}^{\infty} \alpha_{n} x^{n}$. The conditions (3.39) ensure that the series is convergent and imposing (3.29), (3.34) and (3.35) on (3.40), one obtains

$$
\bar{\tau}(\chi)=\tau(\chi), \quad \tau(\chi)^{2}=1, \quad \tau(-\chi)=-\tau(\chi) .
$$

The series representation (3.40) of the matrix $\mathcal{T}$ allows to infer the following remarkable property.

Proposition 3.3 For any solution $\mathcal{R}$ of the boundary Yang-Baxter equation (3.31), $\mathcal{T}$ defined by (3.40) satisfies (3.32), (3.33).

Proof. The statement can be proven in two steps. The first one is to show that the matrix $\mathcal{T}_{\mathcal{R}}(\chi)=\mathcal{R}(\chi) \mathcal{R}(-\chi)$ obeys (3.32) and (3.33), which is done by repeated use of (3.31). The second step is based on the identities

$$
\begin{array}{r}
\mathcal{S}_{12}\left(\chi_{1}, \chi_{2}\right)\left[\mathcal{T}_{2}\left(\chi_{1}\right)\right]^{m} \mathcal{S}_{12}\left(\chi_{2}, \chi_{1}\right)\left[\mathcal{T}_{2}\left(\chi_{2}\right)\right]^{n}= \\
{\left[\mathcal{T}_{2}\left(\chi_{2}\right)\right]^{n} \mathcal{S}_{12}\left(\chi_{1}, \chi_{2}\right)\left[\mathcal{T}_{2}\left(\chi_{1}\right)\right]^{m} \mathcal{S}_{12}\left(\chi_{2}, \chi_{1}\right),} \\
\mathcal{S}_{12}\left(\chi_{1}, \chi_{2}\right)\left[\mathcal{T}_{2}\left(\chi_{1}\right)\right]^{n} \mathcal{S}_{12}\left(\chi_{2}, \chi_{1}\right) \mathcal{R}_{2}\left(\chi_{2}\right)= \\
\mathcal{R}_{2}\left(\chi_{2}\right) \mathcal{S}_{12}\left(\chi_{1},-\chi_{2}\right)\left[\mathcal{T}_{2}\left(\chi_{1}\right)\right]^{n} \mathcal{S}_{12}\left(-\chi_{2}, \chi_{1}\right),
\end{array}
$$

which hold for any integers $m, n \geq 1$ and are the consequence of a recurrent application of (3.32), (3.33).

It is worth mentioning that the above argument makes no use of the values of coefficients $\alpha_{n}$ in (3.40) and the conclusion of proposition 3.3 remains valid for any convergent series in powers of $\mathcal{R}(\chi) \mathcal{R}(-\chi)$.

Summarizing, we have shown that the transmission and transmissionreflection Yang-Baxter equations (3.32) and (3.33) are a consequence of Hermitian analyticity (3.28), (3.29), unitarity (3.11), (3.34), (3.35) and the boundary Yang-Baxter equation (3.31).

We turn now to the Fock representations of $\mathcal{C}_{\mathcal{S}}$. Our goal will be to demonstrate that each doublet $\{\mathcal{R}, \mathcal{T}\}$, satisfying (3.28), (3.29), (3.31), (3.34) and 
(3.35), fully determines a Fock representation $\mathcal{F}_{\mathcal{R}, \mathcal{T}}\left(\mathcal{C}_{\mathcal{S}}\right)$ of $\mathcal{C}_{\mathcal{S}}$. For this purpose we shall construct $\mathcal{F}_{\mathcal{R}, \mathcal{T}}\left(\mathcal{C}_{\mathcal{S}}\right)$ explicitly, extending the projection operator technique developed in [14, 27, 28] for the ZF and boundary algebras, which are particular cases of $\mathcal{C}_{\mathcal{S}}$. The first step is to introduce the $n$-particle subspace $\mathcal{H}^{(n)}$ of $\mathcal{F}_{\mathcal{R}, \mathcal{T}}\left(\mathcal{C}_{\mathcal{S}}\right)$. For this purpose we consider

$$
\mathcal{L}=\bigoplus_{\alpha} L^{2}(\mathbb{R})
$$

equipped with the standard scalar product

$$
(\varphi, \psi)=\int_{-\infty}^{\infty} d \chi \varphi^{\dagger \alpha}(\chi) \psi_{\alpha}(\chi)=\sum_{\alpha} \int_{-\infty}^{\infty} d \chi \bar{\varphi}_{\alpha}(\chi) \psi_{\alpha}(\chi) .
$$

The $n$-particle space $\mathcal{H}^{(n)}$ we are looking for is a subspace of the $n$-fold tensor power $\mathcal{L}^{\otimes n}$, characterized by a suitable projection operator $P^{(n)}$. In order to construct $P^{(n)}$, we proceed as follows. Observing that any element $\varphi \in \mathcal{L}^{\otimes n}$ can be viewed as a column whose entries are $\varphi_{\alpha_{1} \cdots \alpha_{n}}\left(\chi_{1}, \ldots, \chi_{n}\right)$, we define the operators $\left\{\sigma_{i}^{(n)}, \tau^{(n)}: i=1, \ldots, n-1\right\}$ acting on $\mathcal{L}^{\otimes n}$ according to:

$$
\begin{gathered}
{\left[\sigma_{i}^{(n)} \varphi\right]_{\alpha_{1} \ldots \alpha_{n}}\left(\chi_{1}, \ldots, \chi_{i}, \chi_{i+1}, \ldots, \chi_{n}\right)=} \\
{\left[\mathcal{S}_{i i+1}\left(\chi_{i}, \chi_{i+1}\right)\right]_{\alpha_{1} \ldots \alpha_{n}}^{\beta_{1} \ldots \alpha_{n}} \varphi_{\beta_{1} \ldots \beta_{n}}\left(\chi_{1}, \ldots, \chi_{i+1}, \chi_{i}, \ldots, \chi_{n}\right), \quad n \geq 2,} \\
{\left[\tau^{(n)} \varphi\right]_{\alpha_{1} \ldots \alpha_{n}}\left(\chi_{1}, \ldots, \chi_{n}\right)=\mathcal{T}_{\alpha_{n}}^{\beta_{n}}\left(\chi_{n}\right) \varphi_{\alpha_{1} \ldots \alpha_{n-1} \beta_{n}}\left(\chi_{1}, \ldots, \chi_{n-1}, \chi_{n}\right)+} \\
\mathcal{R}_{\alpha_{n}}^{\beta_{n}}\left(\chi_{n}\right) \varphi_{\alpha_{1} \ldots \alpha_{n-1} \beta_{n}}\left(\chi_{1}, \ldots, \chi_{n-1},-\chi_{n}\right), \quad n \geq 1
\end{gathered}
$$

where

$$
\left[\mathcal{S}_{i j}\left(\chi_{i}, \chi_{j}\right)\right]_{\alpha_{1} \ldots \alpha_{n}}^{\beta_{1} \ldots \beta_{n}}=\delta_{\alpha_{1}}^{\beta_{1}} \cdots \widehat{\delta_{\alpha_{i}}^{\beta_{i}}} \cdots \widehat{\delta_{\alpha_{j}}^{\beta_{j}}} \cdots \delta_{\alpha_{n}}^{\beta_{n}} \mathcal{S}_{\alpha_{i} \alpha_{j}}^{\beta_{i} \beta_{j}}\left(\chi_{i}, \chi_{j}\right)
$$

In order to implement eqs. (3.46), (3.47) on the whole $\mathcal{L}^{\otimes n}$, we assume at this stage that the matrix elements $\mathcal{S}_{\alpha_{1} \alpha_{2}}^{\beta_{1} \beta_{2}}\left(\chi_{1}, \chi_{2}\right), \mathcal{T}_{\alpha}^{\beta}(\chi)$ and $\mathcal{R}_{\alpha}^{\beta}(\chi)$ are bounded functions. Now, one can prove

Proposition 3.4 Let $\mathcal{W}_{n}$ be the Weyl group associated with the root systems of the classical Lie algebra $B_{n}$ and let $\left\{\sigma_{i}, \tau: i=1, \ldots, n-1\right\}$ be the generators of $\mathcal{W}_{n}$. The mapping

$$
\phi_{n}: \sigma_{i} \mapsto \sigma_{i}^{(n)}, \quad \phi_{n}: \tau \mapsto \tau^{(n)},
$$


defines a representation of $\mathcal{W}_{n}$ in $\mathcal{L}^{\otimes n}$. Moreover,

$$
P^{(n)} \equiv \frac{1}{2^{n} n !} \sum_{\nu \in \mathcal{W}_{n}} \phi_{n}(\nu)
$$

is an orthogonal projection operator in $\mathcal{L}^{\otimes n}$.

Proof. One has by construction

$$
\begin{array}{cr}
\sigma_{i}^{(n)} \sigma_{j}^{(n)}=\sigma_{j}^{(n)} \sigma_{i}^{(n)}, & |i-j| \geq 2, \\
\sigma_{i}^{(n)} \tau=\tau \sigma_{i}^{(n)}, & 1 \leq i<n-2 .
\end{array}
$$

Using the Yang-Baxter equations (3.12), (3.31)-(3.33), (3.38), one shows that

$$
\begin{aligned}
\sigma_{i}^{(n)} \sigma_{i+1}^{(n)} \sigma_{i}^{(n)} & =\sigma_{i+1}^{(n)} \sigma_{i}^{(n)} \sigma_{i+1}^{(n)}, \\
\sigma_{n-1}^{(n)} \tau \sigma_{n-1}^{(n)} \tau & =\tau \sigma_{n-1}^{(n)} \tau \sigma_{n-1}^{(n)} .
\end{aligned}
$$

The unitarity conditions (3.11) and (3.34), (3.35) imply

$$
\left[\sigma_{i}^{(n)}\right]^{2}=\tau^{2}=\mathbf{1}
$$

Consequently, $\phi_{n}$ is a representation of $\mathcal{W}_{n}$ in $\mathcal{L}^{\otimes n}$ and $P^{(n)}$ is a projection operator. Finally, from Hermitian analyticity (3.17), (3.28), (3.29), one infers that $\left\{\sigma_{i}^{(n)}, \tau^{(n)}: i=1, \ldots, n-1\right\}$ are Hermitian operators. Therefore, $P^{(n)}$ is orthogonal.

We have at this stage enough background to construct the Fock representation $\mathcal{F}_{\mathcal{R}, \mathcal{T}}\left(\mathcal{C}_{\mathcal{S}}\right)$. The $n$-particle space is defined by

$$
\mathcal{H}^{(0)}=\mathbb{C}, \quad \mathcal{H}^{(n)}=P^{(n)} \mathcal{L}^{\otimes n}, \quad n \geq 1,
$$

the total Fock space being

$$
\mathcal{H}=\bigoplus_{n=0}^{\infty} \mathcal{H}^{(n)}
$$

The finite particle space $\mathcal{D}$ is the (complex) linear space of sequences $\varphi=\left(\varphi^{(0)}, \varphi^{(1)}, \ldots, \varphi^{(n)}, \ldots\right)$ with $\varphi^{(n)} \in \mathcal{H}^{(n)}$ and $\varphi^{(n)}=0$ for $n$ large enough. 
$\mathcal{D}$ is dense in $\mathcal{F}_{\mathcal{R}, \mathcal{T}}\left(\mathcal{C}_{\mathcal{S}}\right)$. The vacuum state is $\Omega=(1,0, \ldots, 0, \ldots)$ and belong to $\mathcal{D}$. The smeared bulk operators $\left\{a(f), a^{*}(f): f \in \mathcal{L}\right\}$ act on $\mathcal{D}$ as follows:

$$
\begin{gathered}
a(f) \Omega=0 \\
{[a(f) \varphi]_{\alpha_{1} \cdots \alpha_{n}}^{(n)}\left(\chi_{1}, \ldots, \chi_{n}\right)=\sqrt{n+1} \int_{-\infty}^{\infty} d \chi f^{\dagger \alpha_{0}}(\chi) \varphi_{\alpha_{0} \alpha_{1} \cdots \alpha_{n}}^{(n+1)}\left(\chi, \chi_{1}, \ldots, \chi_{n}\right)} \\
{\left[a^{*}(f) \varphi\right]_{\alpha_{1} \cdots \alpha_{n}}^{(n)}\left(\chi_{1}, \ldots, \chi_{n}\right)=\sqrt{n}\left[P^{(n)} f \otimes \varphi^{(n-1)}\right]_{\alpha_{1} \cdots \alpha_{n}}\left(\chi_{1}, \ldots, \chi_{n}\right)}
\end{gathered}
$$

In general, $a(f)$ and $a^{*}(f)$ are unbounded operators on $\mathcal{D}$. For any $\varphi^{(n)} \in$ $\mathcal{H}^{(n)}$ one has however the estimate

$$
\left\|a^{\natural}(f) \varphi^{(n)}\right\| \leq \sqrt{n}\|f\|\left\|\varphi^{(n)}\right\|,
$$

where $a^{\natural}(f)$ stands for $a(f)$ or $a^{*}(f)$. Therefore $a(f)$ and $a^{*}(f)$ are bounded on each $\mathcal{H}^{(n)}$.

We now turn to the defect generators, defining $t_{\alpha}^{\beta}(\chi)$ and $r_{\alpha}^{\beta}(\chi)$ as the following multiplicative operators on $\mathcal{D}$ :

$$
\begin{aligned}
& {\left[r_{\alpha}^{\beta}(\chi) \varphi\right]_{\gamma_{1} \ldots \gamma_{n}}^{(n)}\left(\chi_{1}, \ldots, \chi_{n}\right)=\left[\mathcal{S}_{01}\left(\chi, \chi_{1}\right) \mathcal{S}_{12}\left(\chi, \chi_{2}\right) \cdots \mathcal{S}_{(n-1) n}\left(\chi, \chi_{n}\right) \mathcal{R}_{n}(\chi)\right.} \\
& \left.\cdot \mathcal{S}_{(n-1) n}\left(\chi_{n},-\chi\right) \cdots \mathcal{S}_{12}\left(\chi_{2},-\chi\right) \mathcal{S}_{01}\left(\chi_{1},-\chi\right)\right]_{\alpha \gamma_{1} \ldots \gamma_{n}}^{\beta \delta_{1} \ldots \delta_{n}} \varphi_{\delta_{1} \ldots \delta_{n}}^{(n)}\left(\chi_{1}, \ldots, \chi_{n}\right) \\
& {\left[t_{\alpha}^{\beta}(\chi) \varphi\right]_{\gamma_{1} \ldots \gamma_{n}}^{(n)}\left(\chi_{1}, \ldots, \chi_{n}\right)=\left[\mathcal{S}_{01}\left(\chi, \chi_{1}\right) \mathcal{S}_{12}\left(\chi, \chi_{2}\right) \cdots \mathcal{S}_{(n-1) n}\left(\chi, \chi_{n}\right) \mathcal{T}_{n}(\chi)\right.} \\
& \left.\quad \mathcal{S}_{(n-1) n}\left(\chi_{n}, \chi\right) \cdots \mathcal{S}_{12}\left(\chi_{2}, \chi\right) \mathcal{S}_{01}\left(\chi_{1}, \chi\right)\right]_{\alpha \gamma_{1} \ldots \gamma_{n}}^{\beta \delta_{1} \ldots \delta_{n}} \varphi_{\delta_{1} \ldots \delta_{n}}^{(n)}\left(\chi_{1}, \ldots, \chi_{n}\right)
\end{aligned}
$$

combined with (3.30). As expected, the defect operators preserve the bulk particle number.

For deriving the commutation properties of (3.59), (3.60), (3.62), (3.63) on $\mathcal{D}$, it is convenient to introduce the operator-valued distributions $a_{\alpha}(\chi)$ and $a^{* \alpha}(\chi)$ defined by

$$
a(f)=\int_{-\infty}^{\infty} d \chi f^{\dagger \alpha}(\chi) a_{\alpha}(\chi), \quad a^{*}(f)=\int_{-\infty}^{\infty} d \chi f_{\alpha}(\chi) a^{* \alpha}(\chi) .
$$

A straightforward computation allows to prove now 
Proposition 3.5 The operator-valued distributions $\left\{a_{\alpha}(\chi), a^{* \alpha}(\chi)\right\}$ and $\left\{r_{\alpha}^{\beta}(\chi), t_{\alpha}^{\beta}(\chi)\right\}$ satisfy the exchange relations (3.1)-(3.10) and the constraints (3.13) and (3.14) on $\mathcal{D}$. The involution $I$ is realized as Hermitian conjugation with respect to the scalar product (3.45).

This result completes the construction of the Fock representation $\mathcal{F}_{\mathcal{R}, \mathcal{T}}\left(\mathcal{C}_{\mathcal{S}}\right)$, which is the basic tool in the physical applications discussed in this paper. Fixing $\mathcal{C}_{\mathcal{S}}, \mathcal{F}_{\mathcal{R}, \mathcal{T}}\left(\mathcal{C}_{\mathcal{S}}\right)$ is indeed fully determined by $\{\mathcal{R}, \mathcal{T}\}$ satisfying eqs. (3.28), (3.29), (3.31)-(3.35). Besides some concrete examples, little is known in general about the solution set of the latter. There exist, however, one particular case of physical importance, which is described in

Proposition 3.6 Suppose that $\mathcal{R}$ obeys

$$
\mathcal{S}_{12}\left(\chi_{1}, \chi_{2}\right) \mathcal{R}_{2}\left(\chi_{1}\right)=\mathcal{R}_{1}\left(\chi_{1}\right) \mathcal{S}_{12}\left(-\chi_{1}, \chi_{2}\right) \text {. }
$$

Then $\mathcal{R}$ satisfies the boundary Yang-Baxter equation (3.31). Moreover, $\mathcal{T}$ obeys

$$
\mathcal{S}_{12}\left(\chi_{1}, \chi_{2}\right) \mathcal{T}_{2}\left(\chi_{1}\right)=\mathcal{T}_{1}\left(\chi_{1}\right) \mathcal{S}_{12}\left(\chi_{1}, \chi_{2}\right),
$$

and

$$
r_{\alpha}^{\beta}(\chi) \varphi=\mathcal{R}_{\alpha}^{\beta}(\chi) \varphi, \quad t_{\alpha}^{\beta}(\chi) \varphi=\mathcal{T}_{\alpha}^{\beta}(\chi) \varphi,
$$

hold for all $\varphi \in \mathcal{D}$.

Proof. Eq. (3.31) follows directly from (3.11) and (3.65). The identity (3.66) is a consequence of (3.65) and the series representation (3.40). Equations (3.67) follow from (3.63), (3.62) and (3.65), (3.66). Note also that (2.17), (2.18) are recovered from (3.25), (3.26) and (3.67).

The condition (3.65) sort of linearizes eqs. (3.31) (3.33) and defines a special subset of representations $\widetilde{\mathcal{F}}\left(\mathcal{C}_{\mathcal{S}}\right) \subset \mathcal{F}\left(\mathcal{C}_{\mathcal{S}}\right)$, whose defect operators are proportional to the identity in $\mathcal{H}$. All Fock representations of the algebras $\mathcal{C}_{B}$ and $\mathcal{C}_{F}$, introduced above in the context of $\delta$-impurities, belong to this subset because (3.65) is identically satisfied for the bosonic and fermionic exchange factors

$$
\mathcal{S}_{\alpha_{1} \alpha_{2}}^{\beta_{1} \beta_{2}}\left(\chi_{1}, \chi_{2}\right)= \pm \delta_{\alpha_{1}}^{\beta_{2}} \delta_{\alpha_{2}}^{\beta_{1}}
$$

Let us observe in this respect that $\mathcal{T}$ and $\mathcal{R}$, given by (2.4), (2.16), obey Hermitian analyticity (3.28, 3.29) and unitarity (3.34, 3.35). 


\section{Factorized scattering with impurities}

We develop in this section a general approach to factorized scattering in $(1+1)$-dimensional integrable models with impurity.

\subsection{Kinematics}

Let $E$ and $p$ be the energy and momentum of any asymptotic bulk particle. Usually $E$ and $p$ are not independent and obey some dispersion relation. The latter can be implemented expressing both $E$ and $p$ in terms of one parameter $\chi \in \mathbb{R}$, namely

$$
E=E(\chi), \quad p=p(\chi)
$$

It is instructive to keep in mind the following two examples:

- Relativistic dispersion relation

$$
E(\chi)=m \cosh (\chi), \quad p(\chi)=m \sinh (\chi),
$$

where $m>0$ is the mass and $\chi$ the rapidity.

- Non-relativistic dispersion relation

$$
E(\chi)=\frac{m \chi^{2}}{2}+U, \quad p(\chi)=m \chi
$$

$\chi$ being the velocity and $U$ some constant.

Note that both of these relations satisfy

$$
\epsilon(p)=\epsilon(\chi)
$$

$\epsilon$ being the sign function. We also observe that a Lorentz boost in (4.2) and a Galilean transformation in (4.3) are both realized by a translation $\chi \mapsto \chi+\alpha$.

In what follows we adopt a dispersion relation (4.1), which satisfies (4.4) but is otherwise generic and parametrizes any asymptotic bulk particle by $\chi \in \mathbb{R}$ and its isotopic index $i=1, \ldots, N$. Assuming that the impurity, localized at $x=0$, has no internal degrees of freedom, and taking into account (4.4), the fundamental building blocks of factorized scattering are:

(i) the two-body bulk scattering matrix $S_{i_{1} i_{2}}^{j_{1} j_{2}}\left(\chi_{1}, \chi_{2}\right)$ defined on $\mathbb{R} \times \mathbb{R}$; 
(ii) the right and left reflection matrices $R^{+j}{ }_{i}(\chi)$ and $R^{-j}(\chi)$, defined on $\mathbb{R}_{+}$

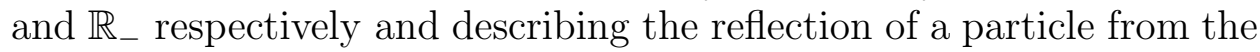
impurity;

(iii) the left and right transmission matrices $T_{i}^{+j}(\chi)$ and $T_{i}^{-j}(\chi)$, defined on $\mathbb{R}_{+}$and $\mathbb{R}_{-}$respectively and describing the transmission of a particle by the impurity.

We emphasize that $S$ is allowed to depend on $\chi_{1}$ and $\chi_{2}$ separately [25], generalizing the previous attempts [15, 16, 19, where $S$ is assumed to depend on $\chi_{1}-\chi_{2}$ only. As already argued in [21, this last condition is too restrictive and quite artificial in the presence of defects. With the dispersion relation (4.2) for instance, $S_{i_{1} i_{2}}^{j_{1} j_{2}}\left(\chi_{1}-\chi_{2}\right)$ turns out to be Lorentz-invariant. But we know that Lorentz symmetry is generally broken by impurities. Accordingly, we allow $S$ to depend on $\chi_{1}$ and $\chi_{2}$ separately. This leads to a natural generalization of the inverse scattering method, which avoids the no-go theorem of [15, 19] and describes a large set of integrable systems, not covered there.

One should also keep in mind that our transmission and reflection matrices are not defined on the whole $\mathbb{R}$, but only for values of $\chi$ in the relative physical kinematic domains specified in (ii) and (iii). This information must be sufficient for reconstructing the total scattering operator $\mathbf{S}$ and we demonstrate below that this is indeed the case.

The data $\left\{S, R^{ \pm}, T^{ \pm}\right\}$are subject to a number of constraints, ensuring physical unitarity of the scattering operator $\mathbf{S}$ and factorization of the transition amplitudes. Let us first concentrate on unitarity. Since integrability implies particle number conservation, the restriction $\mathbf{S}^{(1)}$ of $\mathbf{S}$ to the oneparticle subspace is a well-defined operator. One has (see also eq. (4.49) below)

$$
\mathbf{S}^{(1)}(\chi)=\left(\begin{array}{cc}
R^{+}(\chi) & T^{+}(\chi) \\
T^{-}(-\chi) & R^{-}(-\chi)
\end{array}\right), \quad \chi>0 .
$$

Equation (4.5) reflects an essential difference with respect to any Lorentzinvariant theory, where $\mathbf{S}^{(1)}=\mathbb{I}$ is mandatory [23]. Unitarity

$$
\mathbf{S}^{(1)}(\chi)\left[\mathbf{S}^{(1)}\right]^{\dagger}(\chi)=\left[\mathbf{S}^{(1)}\right]^{\dagger}(\chi) \mathbf{S}^{(1)}(\chi)=\mathbb{I}
$$

implies

$$
\begin{aligned}
& R^{ \pm}( \pm \chi)\left[R^{ \pm}\right]^{\dagger}( \pm \chi)+T^{ \pm}( \pm \chi)\left[T^{ \pm}\right]^{\dagger}( \pm \chi)=\mathbb{I} \\
& {\left[R^{ \pm}\right]^{\dagger}( \pm \chi) R^{ \pm}( \pm \chi)+\left[T^{\mp}\right]^{\dagger}(\mp \chi) T^{\mp}(\mp \chi)=\mathbb{I}}
\end{aligned}
$$




$$
\begin{aligned}
& R^{ \pm}( \pm \chi)\left[T^{\mp}\right]^{\dagger}(\mp \chi)+T^{ \pm}( \pm \chi)\left[R^{\mp}\right]^{\dagger}(\mp \chi)=0 \\
& {\left[R^{ \pm}\right]^{\dagger}( \pm \chi) T^{ \pm}( \pm \chi)+\left[T^{\mp}\right]^{\dagger}(\mp \chi) R^{\mp}(\mp \chi)=0}
\end{aligned}
$$

where $\chi>0$. We stress that (4.7)-(4.10) are necessary and sufficient conditions: any violation of (4.7)-(4.10) breaks down the unitarity of $\mathbf{S}^{(1)}$ and, consequently, of $\mathbf{S}$. It is worth mentioning that in our previous paper [21]

$$
\begin{gathered}
R^{ \pm}( \pm \chi) R^{\mp}(\mp \chi)+T^{ \pm}( \pm \chi) T^{ \pm}( \pm \chi)=\mathbb{I}, \\
R^{ \pm}( \pm \chi) T^{\mp}(\mp \chi)+T^{ \pm}( \pm \chi) R^{ \pm}( \pm \chi)=0, \\
{\left[R^{ \pm}\right]^{\dagger}( \pm \chi)=R^{\mp}(\mp \chi), \quad\left[T^{ \pm}\right]^{\dagger}( \pm \chi)=T^{ \pm}( \pm \chi)}
\end{gathered}
$$

were imposed instead of (4.7)-(4.10). The conditions (4.11)-(4.13) are stronger than (4.7) -(4.10) and provide some technical advantage [21] in dealing with the factorization constraints obtained below. One can easily see however that $\delta$-type defects (see e.g. eq. (2.4)) violate ${ }^{*}$ (4.11)-(4.13). For this reason we avoid the use of (4.11)-(4.13) in the present paper, keeping (4.7)-(4.10) which are respected by the $\delta$-impurities described in sect. 2. We conclude the issue recalling that bulk scattering unitarity is controlled by

$$
S_{12}\left(\chi_{1}, \chi_{2}\right) S_{12}\left(\chi_{2}, \chi_{1}\right)=\mathbb{I}, \quad S_{12}^{\dagger}\left(\chi_{1}, \chi_{2}\right)=S_{12}\left(\chi_{2}, \chi_{1}\right) .
$$

For analysing the constraints following from factorization, it is convenient to display the data $\left\{S, R^{ \pm}, T^{ \pm}\right\}$graphically. This is done in Fig. 1, where the time is flowing along the vertical direction, single lines denote the particle world lines and the double line is the impurity. Requiring factorization of all possible three-body processes leads to a series of relations among $S, T^{ \pm}$ and $R^{ \pm}$. As is well-known [2], the scattering of three particles implies the quantum Yang-Baxter equation

$$
S_{12}\left(\chi_{1}, \chi_{2}\right) S_{23}\left(\chi_{1}, \chi_{3}\right) S_{12}\left(\chi_{2}, \chi_{3}\right)=S_{23}\left(\chi_{2}, \chi_{3}\right) S_{12}\left(\chi_{1}, \chi_{3}\right) S_{23}\left(\chi_{1}, \chi_{2}\right),
$$

whose graphic representation is familiar and is omitted for conciseness.

The consistency conditions stemming from the scattering of two particles between themselves and the impurity, can be organized in three groups.

(a) Pure reflection:

$$
S_{12}\left(\chi_{1}, \chi_{2}\right) R_{2}^{+}\left(\chi_{1}\right) S_{12}\left(\chi_{2},-\chi_{1}\right) R_{2}^{+}\left(\chi_{2}\right)=
$$

\footnotetext{
${ }^{*}$ Correspondence with O. A. Castro-Alvaredo and A. Fring on this point is kindly acknowledged.
} 

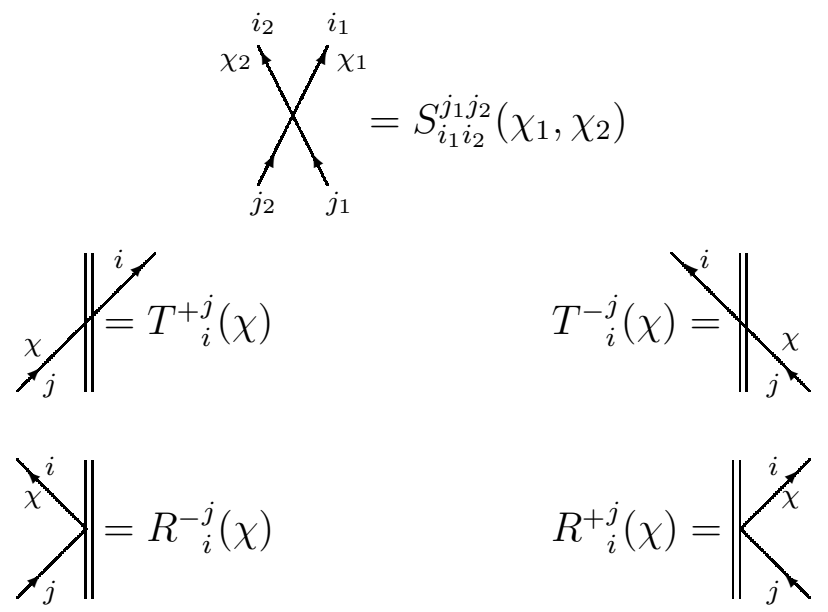

Figure 1: The two-body processes.

$$
\begin{gathered}
R_{2}^{+}\left(\chi_{2}\right) S_{12}\left(\chi_{1},-\chi_{2}\right) R_{2}^{+}\left(\chi_{1}\right) S_{12}\left(-\chi_{2},-\chi_{1}\right) \\
S_{12}\left(\chi_{1}, \chi_{2}\right) R_{1}^{-}\left(\chi_{2}\right) S_{12}\left(-\chi_{2}, \chi_{1}\right) R_{1}^{-}\left(\chi_{1}\right)= \\
R_{1}^{-}\left(\chi_{1}\right) S_{12}\left(-\chi_{1}, \chi_{2}\right) R_{1}^{-}\left(\chi_{2}\right) S_{12}\left(-\chi_{2},-\chi_{1}\right) .
\end{gathered}
$$

Equations (4.16) and (4.17) concern the reflection on $\mathbb{R}_{+}$and $\mathbb{R}_{-}$respectively. Using the rules in Fig. 1 and moving back in time, one gets the graphic representation of (4.16) shown in Fig. 2.

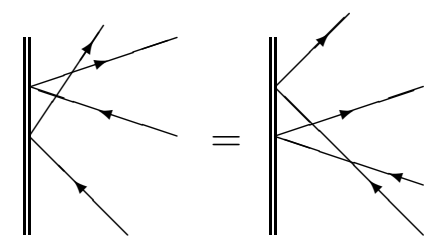

Figure 2: Pure reflection.

The picture associated to (4.17) is obtained from Fig. 2 by reflection with respect to the impurity world line.

(b) Pure transmission:

$$
\begin{aligned}
& T_{1}^{+}\left(\chi_{1}\right) S_{12}\left(\chi_{1}, \chi_{2}\right) T_{1}^{-}\left(\chi_{2}\right)=T_{2}^{-}\left(\chi_{2}\right) S_{12}\left(\chi_{1}, \chi_{2}\right) T_{2}^{+}\left(\chi_{1}\right), \\
& S_{12}\left(\chi_{1}, \chi_{2}\right) T_{1}^{-}\left(\chi_{2}\right) T_{2}^{-}\left(\chi_{1}\right)=T_{1}^{-}\left(\chi_{1}\right) T_{2}^{-}\left(\chi_{2}\right) S_{12}\left(\chi_{1}, \chi_{2}\right), \\
& S_{12}\left(\chi_{1}, \chi_{2}\right) T_{1}^{+}\left(\chi_{2}\right) T_{2}^{+}\left(\chi_{1}\right)=T_{1}^{+}\left(\chi_{1}\right) T_{2}^{+}\left(\chi_{2}\right) S_{12}\left(\chi_{1}, \chi_{2}\right) .
\end{aligned}
$$


Equations (4.18) and (4.19) are represented in Fig. 3(a) and Fig. 3(b) respectively.

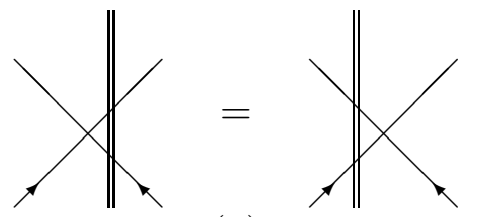

(a)

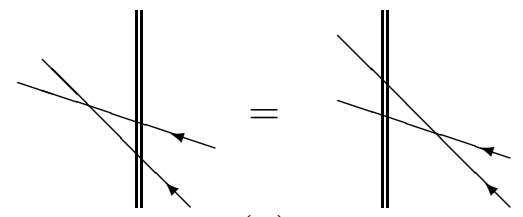

(b)

Figure 3: Pure transmission.

As before, the picture corresponding to eq. (4.20) is obtained from Fig. 3(b) by reflection.

(c) Mixed relations:

$$
\begin{aligned}
& R_{1}^{+}\left(\chi_{1}\right) T_{2}^{-}\left(\chi_{2}\right)=T_{2}^{-}\left(\chi_{2}\right) S_{12}\left(\chi_{1}, \chi_{2}\right) R_{2}^{+}\left(\chi_{1}\right) S_{12}\left(\chi_{2},-\chi_{1}\right) \\
& T_{1}^{+}\left(\chi_{1}\right) R_{2}^{-}\left(\chi_{2}\right)=T_{1}^{+}\left(\chi_{1}\right) S_{12}\left(\chi_{1}, \chi_{2}\right) R_{1}^{-}\left(\chi_{2}\right) S_{12}\left(-\chi_{2}, \chi_{1}\right) \\
& R_{1}^{+}\left(\chi_{1}\right) T_{2}^{+}\left(\chi_{2}\right)=S_{12}\left(\chi_{1}, \chi_{2}\right) R_{2}^{+}\left(\chi_{1}\right) S_{12}\left(\chi_{2},-\chi_{1}\right) T_{2}^{+}\left(\chi_{2}\right) \\
& T_{1}^{-}\left(\chi_{1}\right) R_{2}^{-}\left(\chi_{2}\right)=S_{12}\left(\chi_{1}, \chi_{2}\right) R_{1}^{-}\left(\chi_{2}\right) S_{12}\left(-\chi_{2}, \chi_{1}\right) T_{1}^{-}\left(\chi_{1}\right) \\
& R_{1}^{+}\left(\chi_{1}\right) T_{2}^{-}\left(\chi_{2}\right) S_{12}\left(-\chi_{1}, \chi_{2}\right)=T_{2}^{-}\left(\chi_{2}\right) S_{12}\left(\chi_{1}, \chi_{2}\right) R_{2}^{+}\left(\chi_{1}\right) \\
& T_{1}^{+}\left(\chi_{1}\right) R_{2}^{-}\left(\chi_{2}\right) S_{12}\left(\chi_{1},-\chi_{2}\right)=T_{1}^{+}\left(\chi_{1}\right) S_{12}\left(\chi_{1}, \chi_{2}\right) R_{1}^{-}\left(\chi_{2}\right) \\
& R_{2}^{+}\left(\chi_{1}\right) S_{12}\left(\chi_{2},-\chi_{1}\right) T_{2}^{+}\left(\chi_{2}\right)=S_{12}\left(\chi_{2}, \chi_{1}\right) R_{1}^{+}\left(\chi_{1}\right) T_{2}^{+}\left(\chi_{2}\right) \\
& R_{1}^{-}\left(\chi_{2}\right) S_{12}\left(-\chi_{2}, \chi_{1}\right) T_{1}^{-}\left(\chi_{1}\right)=S_{12}\left(\chi_{2}, \chi_{1}\right) T_{1}^{-}\left(\chi_{1}\right) R_{2}^{-}\left(\chi_{2}\right)
\end{aligned}
$$

Equations (4.21) and (4.25) are shown in Fig. 4(a) and 4(b) respectively, whereas eqs. (4.23) and (4.27) are drawn in Fig. 4(c) and 4(d). The pictures related to the remaining four mixed equations are obtained from Fig. 4 by reflection, which completes the description of all three-body processes.

Summarizing, the scattering data $\left\{S, R^{ \pm}, T^{ \pm}\right\}$are required to satisfy two sets of conditions: unitarity constraints (4.7)-(4.10), (4.14) and factorization constraints (4.15)-(4.28). The general solution of this long list of matrix equations is currently unknown. In order to simplify the problem, we consider invertible $T^{ \pm}$. From eqs. (4.25) and (4.28) one then infers that

$$
S_{12}\left(\chi_{1}, \chi_{2}\right) R_{2}^{ \pm}\left(\chi_{1}\right)=R_{1}^{ \pm}\left(\chi_{1}\right) S_{12}\left(-\chi_{1}, \chi_{2}\right),
$$

which implies the validity of all (4.16), (4.17), (4.21)-(4.28). Therefore, assuming that $T^{ \pm}$are invertible, one is left with eqs. (4.7)-(4.10), (4.14), 


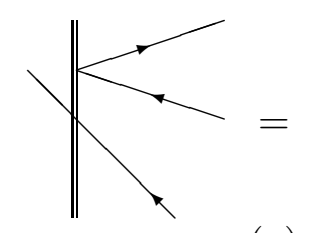

(a)

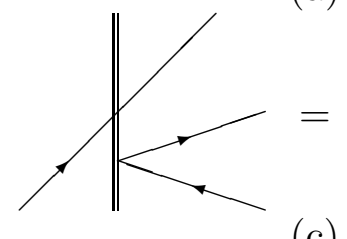

(c)
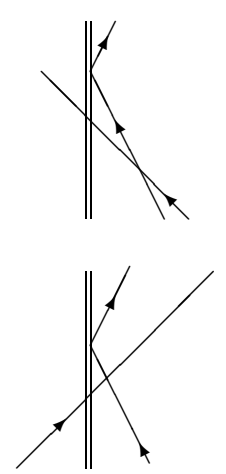

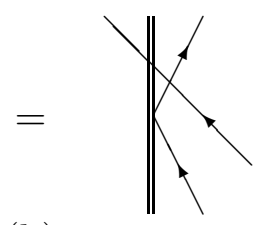

(b)

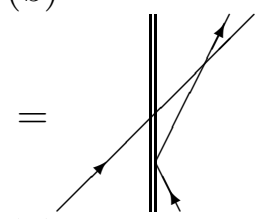

(d)

Figure 4: Mixed relations.

(4.15), (4.18)-(4.20) and (4.29), which simplifies a little the problem of deriving explicit solutions $\left\{S, R^{ \pm}, T^{ \pm}\right\}$. At this stage, it might be useful to give some examples, starting with the $g l(N)$-invariant $S$-matrix

$$
S_{12}\left(\chi_{1}, \chi_{2}\right)=\frac{1}{s\left(\chi_{1}\right)-s\left(\chi_{2}\right)+i g}\left\{\left[s\left(\chi_{1}\right)-s\left(\chi_{2}\right)\right] P_{12}+i g \mathbb{I} \otimes \mathbb{I}\right\},
$$

where $P_{12}$ is the standard flip operator, $g \in \mathbb{R}$, and $s(\chi)$ is any real valued even function. For $R^{ \pm}$and $T^{ \pm}$one derives

$$
\begin{gathered}
R^{ \pm}(\chi)=[\cos p( \pm \chi)] \exp \left[i q^{ \pm}( \pm \chi)\right] \mathbb{I} \\
T^{ \pm}(\chi)= \pm[\sin p( \pm \chi)] \exp \left[i q^{ \pm}( \pm \chi)\right] \mathbb{I},
\end{gathered}
$$

$p(\chi)$ and $q^{ \pm}(\chi)$ being arbitrary real valued functions on $\mathbb{R}_{+}$. In this example both reflection and transmission preserve the isotopic type and all isotopic types have the same reflection and transmission amplitude.

A more complicated example is provided by the Toda type $S$-matrix

$$
S_{i_{1} i_{2}}^{j_{1} j_{2}}\left(\chi_{1}, \chi_{2}\right)=\exp \left[i s_{i_{1} i_{2}}\left(\chi_{1}, \chi_{2}\right)\right] \delta_{i_{1}}^{j_{2}} \delta_{i_{2}}^{j_{1}},
$$

where $s_{i_{1} i_{2}}\left(\chi_{1}, \chi_{2}\right)$ are real valued functions obeying

$$
s_{i_{1} i_{2}}\left(\chi_{1}, \chi_{2}\right)=-s_{i_{2} i_{1}}\left(\chi_{2}, \chi_{1}\right), \quad s_{i_{1} i_{2}}\left(\chi_{1}, \chi_{2}\right)=s_{i_{1} i_{2}}\left(\chi_{1},-\chi_{2}\right) .
$$

When $s_{i_{1} i_{2}}\left(\chi_{1}, \chi_{2}\right)$ satisfy (4.34) but are otherwise generic, one finds

$$
\left[R^{ \pm}\right]_{i}^{j}(\chi)=\left[\cos p_{i}( \pm \chi)\right] \exp \left[i q_{i}^{ \pm}( \pm \chi)\right] \delta_{i}^{j}
$$




$$
\left[T^{ \pm}\right]_{i}^{j}(\chi)= \pm\left[\sin p_{i}( \pm \chi)\right] \exp \left[i q_{i}^{ \pm}( \pm \chi)\right] \delta_{i}^{j}
$$

where $p_{i}(\chi)$ and $q_{i}^{ \pm}(\chi)$ are real valued functions on $\mathbb{R}_{+}$. Also here the impurity interaction preserves the isotopic type, but the individual reflection and transmission amplitudes may be different. Finally, if some of the entries $s_{i_{1} i_{2}}\left(\chi_{1}, \chi_{2}\right)$ coincide, non-diagonal elements in $R^{ \pm}$and $T^{ \pm}$are allowed [21] and the isotopic type is not preserved.

\subsection{Scattering operator and transition amplitudes}

We have so far described in great detail the main features of the physical data $\left\{S, R^{ \pm}, T^{ \pm}\right\}$for factorized scattering with impurity. The next step is to identify the RT algebra $\mathcal{C}_{\mathcal{S}}$ and its Fock representation $\mathcal{F}_{\mathcal{R}, \mathcal{T}}\left(\mathcal{C}_{\mathcal{S}}\right)$ producing the total scattering operator $\mathbf{S}$ and the transition amplitudes, corresponding to $\left\{S, R^{ \pm}, T^{ \pm}\right\}$. For this purpose we set

$$
\begin{gathered}
\mathcal{S}\left(\chi_{1}, \chi_{2}\right)=\left(\begin{array}{cccc}
S\left(\chi_{1}, \chi_{2}\right) & 0 & 0 & 0 \\
0 & 0 & S\left(\chi_{1}, \chi_{2}\right) & 0 \\
0 & S\left(\chi_{1}, \chi_{2}\right) & 0 & 0 \\
0 & 0 & 0 & S\left(\chi_{1}, \chi_{2}\right)
\end{array}\right), \quad(4.37) \\
\mathcal{R}(\chi)=\theta(\chi)\left(\begin{array}{cc}
R^{+}(\chi) & 0 \\
0 & {\left[R^{-}\right]^{\dagger}(-\chi)}
\end{array}\right)+\theta(-\chi)\left(\begin{array}{cc}
{\left[R^{+}\right]^{\dagger}(-\chi)} & 0 \\
0 & R^{-}(\chi)
\end{array}\right), \\
\mathcal{T}(\chi)=\theta(\chi)\left(\begin{array}{cc}
0 & {\left[T^{-}\right]^{\dagger}(\chi)} \\
0 & T^{+}(\chi) \\
{\left[T^{+}\right]^{\dagger}(\chi)} & 0
\end{array}\right)+\theta(-\chi)\left(\begin{array}{c}
0 \\
T^{-}(\chi)
\end{array}\right. \\
0
\end{gathered}
$$

$\mathcal{S}, \mathcal{R}$ and $\mathcal{T}$ defined above are admissible because of

Proposition 4.1 The constraints (4.7)-(4.10), (4.14), (4.15) and (4.29) on the data $\left\{S, R^{ \pm}, T^{ \pm}\right\}$imply the validity of (3.11), (3.12), (3.17), (3.28), (3.29), (3.34), (3.35), (3.65) for $\{\mathcal{S}, \mathcal{R}, \mathcal{T}\}$.

Proof. We first observe that the condition of Hermitian analyticity (3.28), $\overline{(3.29)}$ for $\mathcal{T}$ and $\mathcal{R}$ is satisfied by construction. The remaining conditions can be checked by direct computation.

Thus, eq. (4.37) determines the algebra $\mathcal{C}_{\mathcal{S}}$, whereas (4.38), (4.39) fix the representation $\mathcal{F}_{\mathcal{R}, \mathcal{T}}\left(\mathcal{C}_{\mathcal{S}}\right)$ in terms of $\left\{R^{ \pm}, T^{ \pm}\right\}$. We stress that eq. (4.29) 
implies (3.65). Therefore, according to proposition 3.6, $\mathcal{F}_{\mathcal{R}, \mathcal{T}}\left(\mathcal{C}_{\mathcal{S}}\right) \in \widetilde{\mathcal{F}}\left(\mathcal{C}_{\mathcal{S}}\right)$. In other words, the factorization conditions derived in sect. 4.1 select representations from the subclass $\widetilde{\mathcal{F}}\left(\mathcal{C}_{\mathcal{S}}\right) \subset \mathcal{F}\left(\mathcal{C}_{\mathcal{S}}\right)$.

The asymptotic states in $\mathcal{F}_{\mathcal{R}, \mathcal{T}}\left(\mathcal{C}_{\mathcal{S}}\right)$ are defined in complete analogy with the $\delta$-impurity case, discussed in sect. 2. The presence of internal degrees of freedom can be dealt with in a straightforward way. In-states are created from the vacuum by $\left\{a^{*(-, i)}(g): \operatorname{supp} g \subset \mathbb{R}_{+}\right\}$and $\left\{a^{*(+, i)}(g): \operatorname{supp} g \subset \mathbb{R}_{-}\right\}$. The out-states are generated instead by $\left\{a^{*(-, j)}(h): \operatorname{supp} h \subset \mathbb{R}_{-}\right\}$and $\left\{a^{*(+, j)}(h): \operatorname{supp} h \subset \mathbb{R}_{+}\right\}$. By means of (3.2), one can also order the creation operators according to the values of the spectral parameter, using the relation $\prec$ introduced in sect. 2 . We thus define the "in"-coming states by

$$
\left|g_{1}, i_{1} ; \ldots ; g_{m}, i_{m}\right\rangle^{\text {in }}=a^{*\left(\xi_{1}, i_{1}\right)}\left(g_{1}\right) \cdots a^{*\left(\xi_{m}, i_{m}\right)}\left(g_{m}\right) \Omega
$$

where

$$
g_{1} \prec \cdots \prec g_{m}, \quad \xi_{i}= \begin{cases}+, & \operatorname{supp} g_{i} \subset \mathbb{R}_{-}, \\ -, & \operatorname{supp} g_{i} \subset \mathbb{R}_{+} .\end{cases}
$$

The "out"-going states are given by

$$
{ }^{\text {out }}\left\langle h_{1}, j_{1} ; \ldots ; h_{n}, j_{n}\right|=a^{*\left(\eta_{1}, j_{1}\right)}\left(h_{1}\right) \cdots a^{*\left(\eta_{n}, j_{n}\right)}\left(h_{n}\right) \Omega
$$

with

$$
h_{n} \prec \cdots \prec h_{1}, \quad \eta_{j}= \begin{cases}+, & \operatorname{supp} h_{j} \subset \mathbb{R}_{+}, \\ -, & \operatorname{supp} h_{j} \subset \mathbb{R}_{-} .\end{cases}
$$

The asymptotic spaces $\mathcal{H}^{\text {in }}$ and $\mathcal{H}^{\text {out }}$ are generated by finite linear combinations of vectors of the type (4.40) and (4.42) respectively. Each of these spaces is dense in $\mathcal{H}$. The total scattering operator $\mathbf{S}: \mathcal{H}^{\text {out }} \rightarrow \mathcal{H}^{\text {in }}$ is defined by

$$
\mathbf{S}: a^{*\left(\eta_{1}, j_{1}\right)}\left(h_{1}\right) \cdots a^{*\left(\eta_{n}, j_{n}\right)}\left(h_{n}\right) \Omega \longmapsto a^{*\left(\widetilde{\eta}_{1}, j_{1}\right)}\left(\widetilde{h}_{1}\right) \cdots a^{*\left(\widetilde{\eta}_{n}, j_{n}\right)}\left(\widetilde{h}_{n}\right) \Omega,
$$

where

$$
\widetilde{h}_{k}(\chi)=h_{k}(-\chi), \quad \widetilde{\eta}_{k}=-\eta_{k} .
$$

Using the non-overlapping conditions (4.41), (4.43), it is not difficult to check that

$$
\left(\mathbf{S} \Psi^{\text {out }}, \mathbf{S} \Phi^{\text {out }}\right)=\left(\Psi^{\text {out }}, \Phi^{\text {out }}\right), \quad \forall \Psi^{\text {out }}, \Phi^{\text {out }} \in \mathcal{H}^{\text {out }}
$$

Generalizing the argument of [14, 25], we deduce from (4.44), (4.46) that $\mathbf{S}$ is unitary. 
A generic scattering amplitude reads

$$
\begin{gathered}
{ }^{\text {out }}\left\langle h_{1}, j_{1} ; \ldots ; h_{n}, j_{n} \mid g_{1}, i_{1} ; \ldots ; g_{m}, i_{m}\right\rangle^{\text {in }}= \\
\left(a^{*\left(j_{1}, \eta_{1}\right)}\left(h_{1}\right) \cdots a^{*\left(j_{n}, \eta_{n}\right)}\left(h_{n}\right) \Omega, a^{*\left(i_{1}, \xi_{1}\right)}\left(g_{1}\right) \cdots a^{*\left(i_{m}, \xi_{m}\right)}\left(g_{m}\right) \Omega\right)
\end{gathered}
$$

and can be computed by means of the exchange relation (3.3) and the identities (3.30). The Fock structure implies that (4.47) vanishes unless $m=n$, showing the absence of particle production as expected from integrability. The one-particle transition amplitudes can be deduced from the correlation function

$$
\begin{gathered}
\left(a^{* \beta}(\chi) \Omega, a^{* \alpha}(\varphi) \Omega\right)= \\
{\left[\delta_{\beta}^{\alpha}+\mathcal{T}_{\beta}^{\alpha}(\chi)\right] \delta(\chi-\varphi)+\mathcal{R}_{\beta}^{\alpha}(\chi) \delta(\chi+\varphi) .}
\end{gathered}
$$

One gets

$$
{ }^{\text {out }}\langle h, j \mid g, i\rangle^{\text {in }}= \begin{cases}\int_{0}^{\infty} d \chi \bar{h}(\chi) T_{j}^{+i}(\chi) g(\chi), & \xi=-, \eta=+, \\ \int_{0}^{\infty} d \chi \bar{h}(-\chi) T_{j}^{-i}(-\chi) g(-\chi), & \xi=+, \eta=-, \\ \int_{0}^{\infty} d \chi \bar{h}(\chi) R^{+i}(\chi) g(-\chi), & \xi=+, \eta=+, \\ \int_{0}^{\infty} d \chi \bar{h}(-\chi) R^{-i}(-\chi) g(\chi), & \xi=-, \eta=-,\end{cases}
$$

which describe the particle-impurity interaction and precisely reproduce the one-particle scattering matrix $\mathbf{S}^{(1)}$ given by eq. (4.5).

The particle-particle interaction shows up in the two-particle amplitudes, which can be derived from the correlator

$$
\begin{aligned}
&\left(a^{* \beta_{1}}\left(\chi_{1}\right) a^{* \beta_{2}}\left(\chi_{2}\right) \Omega, a^{* \alpha_{1}}\left(\varphi_{1}\right) a^{* \alpha_{2}}\left(\varphi_{2}\right) \Omega\right)= \\
& \quad\left[\delta_{\beta_{2}}^{\mu}+\mathcal{T}_{\beta_{2}}^{\mu}\left(\chi_{2}\right)\right] \mathcal{S}_{\beta_{1} \mu}^{\alpha_{1} \nu}\left(\chi_{1}, \chi_{2}\right)\left[\delta_{\nu}^{\alpha_{2}}+\mathcal{T}_{\nu}^{\alpha_{2}}\left(\chi_{1}\right)\right] \delta\left(\chi_{1}-\varphi_{2}\right) \delta\left(\chi_{2}-\varphi_{1}\right) \\
& \quad+\mathcal{R}_{\beta_{2}}^{\mu}\left(\chi_{2}\right) \mathcal{S}_{\beta_{1} \mu}^{\alpha_{1}}\left(\chi_{1},-\chi_{2}\right)\left[\delta_{\nu}^{\alpha_{2}}+\mathcal{T}_{\nu}^{\alpha_{2}}\left(\chi_{1}\right)\right] \delta\left(\chi_{1}-\varphi_{2}\right) \delta\left(\chi_{2}+\varphi_{1}\right) \\
& \quad+\left[\delta_{\beta_{2}}^{\mu}+\mathcal{T}_{\beta_{2}}^{\mu}\left(\chi_{2}\right)\right] \mathcal{S}_{\beta_{1} \mu}^{\alpha_{1} \nu}\left(\chi_{1}, \chi_{2}\right) \mathcal{R}_{\nu}^{\alpha_{2}}\left(\chi_{1}\right) \delta\left(\chi_{1}+\varphi_{2}\right) \delta\left(\chi_{2}-\varphi_{1}\right) \\
& \quad+\mathcal{R}_{\beta_{2}}^{\mu}\left(\chi_{2}\right) \mathcal{S}_{\beta_{1} \mu}^{\alpha_{1} \nu}\left(\chi_{1},-\chi_{2}\right) \mathcal{R}_{\nu}^{\alpha_{2}}\left(\chi_{1}\right) \delta\left(\chi_{1}+\varphi_{2}\right) \delta\left(\chi_{2}+\varphi_{1}\right) \\
& \quad+\left[\delta_{\beta_{1}}^{\alpha_{1}}+\mathcal{T}_{\beta_{1}}^{\alpha_{1}}\left(\chi_{1}\right)\right]\left[\delta_{\beta_{2}}^{\alpha_{2}}+\mathcal{T}_{\beta_{2}}^{\alpha_{2}}\left(\chi_{2}\right)\right] \delta\left(\chi_{1}-\varphi_{1}\right) \delta\left(\chi_{2}-\varphi_{2}\right) \\
& \quad+\left[\delta_{\beta_{1}}^{\alpha_{1}}+\mathcal{T}_{\beta_{1}}^{\alpha_{1}}\left(\chi_{1}\right)\right] \mathcal{R}_{\beta_{2}}^{\alpha_{2}}\left(\chi_{2}\right) \delta\left(\chi_{1}-\varphi_{1}\right) \delta\left(\chi_{2}+\varphi_{2}\right) \\
& \quad+ \mathcal{R}_{\beta_{1}}^{\alpha_{1}}\left(\chi_{1}\right)\left[\delta_{\beta_{2}}^{\alpha_{2}}+\mathcal{T}_{\beta_{2}}^{\alpha_{2}}\left(\chi_{2}\right)\right] \delta\left(\chi_{1}+\varphi_{1}\right) \delta\left(\chi_{2}-\varphi_{2}\right) \\
& \quad+ \mathcal{R}_{\beta_{1}}^{\alpha_{1}}\left(\chi_{1}\right) \mathcal{R}_{\beta_{2}}^{\alpha_{2}}\left(\chi_{2}\right) \delta\left(\chi_{1}+\varphi_{1}\right) \delta\left(\chi_{2}+\varphi_{2}\right) .
\end{aligned}
$$


Take for instance the asymptotic states

$$
\begin{array}{cc}
\left|g_{1}, i_{1} ; g_{2}, i_{2}\right\rangle^{\text {in }}, & \xi_{1}=-, \quad \xi_{2}=+, \\
{ }_{\text {out }}\left\langle h_{1}, j_{1} ; h_{2}, j_{2}\right|, & \eta_{1}=+, \quad \eta_{2}=+.
\end{array}
$$

The corresponding transition amplitude receives contributions only from the second and the third term in the right-hand side of (4.50). One finds

$$
\begin{gathered}
{ }_{\text {out }}\left\langle h_{1}, j_{1} ; h_{2}, j_{2} \mid g_{1}, i_{1} ; g_{2}, i_{2}\right\rangle^{\text {in }}= \\
\int_{0}^{\infty} d \chi_{1} d \chi_{2} \bar{h}_{1}\left(\chi_{1}\right) \bar{h}_{2}\left(\chi_{2}\right)\left[R_{j_{2}}^{+k}\left(\chi_{2}\right) S_{j_{1} k}^{i_{1} l}\left(\chi_{1},-\chi_{2}\right) T_{l}^{+i_{2}}\left(\chi_{1}\right) g_{1}\left(-\chi_{2}\right) g_{2}\left(\chi_{1}\right)+\right. \\
\left.R_{j_{1}}^{+i_{1}}\left(\chi_{1}\right) T_{j_{2}}^{+i_{2}}\left(\chi_{2}\right) g_{1}\left(-\chi_{1}\right) g_{2}\left(\chi_{2}\right)\right] .
\end{gathered}
$$

The associated scattering processes are displayed in Fig. 4 (c,d). All possible kinematic domains, respecting the non-overlapping conditions (4.41), (4.43), give rise to nine different two-particle transition amplitudes, which are reported in [21].

Summarizing, the physical scattering data $\left\{S, R^{ \pm}, T^{ \pm}\right\}$determine both the RT algebra $\mathcal{C}_{\mathcal{S}}$ and its Fock representation $\mathcal{F}_{\mathcal{R}, \mathcal{T}}\left(\mathcal{C}_{\mathcal{S}}\right)$ entering the derivation of the S-matrix amplitudes. The asymptotic states are obtained by acting with the particle creation operators on the standard Fock vacuum $\Omega \in \mathcal{F}_{\mathcal{R}, \mathcal{T}}\left(\mathcal{C}_{\mathcal{S}}\right)$. It is worth stressing that our scheme makes no use of any auxiliary construction of a boundary state with prescribed reflection and transmission properties. This essential difference with respect to all previous approaches of the subject [11]-[20] represents a relevant theoretical and technical advantage of the framework based on the RT algebra $\mathcal{C}_{\mathcal{S}}$.

We emphasize, in conclusion, that the above scattering theory is based entirely on the data $\left\{S, R^{ \pm}, T^{ \pm}\right\}$for real values of the spectral parameter $\chi$. For this reason the results of this work are very general and remain valid also after imposing all physical conditions (such as crossing symmetry and certain meromorphic structure) on the continuation of $\left\{S, R^{ \pm}, T^{ \pm}\right\}$to the complex $\chi$-plane.

\section{Conclusions and perspectives}

We developed in this article a framework for dealing with factorized scattering from reflecting and transmitting impurities in $1+1$ dimensions. Our starting point was the analysis of some exactly solvable models with $\delta$-type impurities, 
which led us directly to the main tool of our approach, the RT algebra $\mathcal{C}_{\mathcal{S}}$. The interaction of a particle with the impurity is implemented in $\mathcal{C}_{\mathcal{S}}$ by the reflection and transmission generators $\left\{r_{\alpha}^{\beta}(\chi)\right\}$ and $\left\{t_{\alpha}^{\beta}(\chi)\right\}$ respectively. As already mentioned, setting $t_{\alpha}^{\beta}(\chi)=0$, one gets from $\mathcal{C}_{\mathcal{S}}$ another useful algebra $\mathcal{B}_{\mathcal{S}}$, which describes 14 factorized scattering from a purely reflecting boundary. In this context $\mathcal{B}_{\mathcal{S}}$ applies also to the construction of off-shell correlation functions [29, 24] and to the study of symmetries [30]. Moreover, setting $r_{\alpha}^{\beta}(\chi)=t_{\alpha}^{\beta}(\chi)=0$, one obtains the celebrated ZF algebra. Therefore, $\mathcal{C}_{\mathcal{S}}$ indeed represents a universal structure for dealing with integrable models in $1+1$ dimensions.

The Fock representations of $\mathcal{C}_{\mathcal{S}}$ also exhibit remarkable features. The operators $\left\{r_{\alpha}^{\beta}(\chi), t_{\alpha}^{\beta}(\chi)\right\}$ condense in the vacuum $\Omega \in \mathcal{F}_{\mathcal{R}, \mathcal{T}}\left(\mathcal{C}_{\mathcal{S}}\right)$. The relative condensates $\left\{\mathcal{R}_{\alpha}^{\beta}(\chi), \mathcal{T}_{\alpha}^{\beta}(\chi)\right\}$ are directly related to the physical reflection and transmission amplitudes. There is no need for special boundary or reflection-transmission states in our scheme. The use of the standard Fock vacuum $\Omega \in \mathcal{F}_{\mathcal{R}, \mathcal{T}}\left(\mathcal{C}_{\mathcal{S}}\right)$ for deriving the asymptotic states significantly simplifies the construction.

We established a complete set of factorization conditions for scattering with impurities in $1+1$ dimensions, showing that they admit solutions with non-trivial bulk scattering if the requirement of Lorentz (Galilean) invariance on the bulk $S$-matrix is relaxed. This feature, which is not surprising in the presence of defects, allows the no-go theorem of [19] to be avoided.

The concept of RT algebra, introduced in this paper, opens a variety of new directions for further research. On the mathematical side, the interplay between $\mathcal{C}_{\mathcal{S}}, \mathcal{B}_{\mathcal{S}}$ and $\mathrm{ZF}$ algebras deserves a more detailed analysis. A link between $\mathcal{B}_{\mathcal{S}}$ and the $\mathrm{ZF}$ algebra has been explored in [31. From the physical point of view, $\mathcal{C}_{\mathcal{S}}$ appears to be the natural candidate for replacing the $\mathrm{ZF}$ algebra in the form-factor program for integrable models with impurities. The construction of off-shell local fields in this context is a challenging open problem. We strongly believe that besides to integrable systems, RT algebras apply also to $(1+1)$-dimensional conformal field theory with permeable walls, which partly transmit and partly reflect the incident waves. Such theories [32, 33] have obvious relevance to critical phenomena and have recently acquired some importance in the theory of strings and branes. Finally, for applications to impurity problems in condensed-matter physics, one needs finite temperature representations of the RT algebras, which requires the construction of Kubo-Martin-Schwinger states over $\mathcal{C}_{\mathcal{S}}$. We are currently investigating some of these issues. 


\section{References}

[1] C. N. Yang, Phys. Rev. Lett. 19 (1967) 1312.

[2] A. B. Zamolodchikov and A. B. Zamolodchikov, Ann. Phys. (N.Y.) 120 (1979) 253.

[3] L. D. Faddeev, E. K. Sklyanin and L. A. Takhtajan, Theor. Math. Phys. 40 (1980) 688 [Teor. Mat. Fiz. 40 (1979) 194].

[4] L. D. Faddeev, Sov. Sci. Rev. C 1 (1980) 107.

[5] M. Karowski and P. Weisz, Nucl. Phys. B 139 (1978) 455.

[6] F. A. Smirnov, Form-factors in completely integrable models of quantum field theory (World Scientific, Singapore, 1992).

[7] I. V. Cherednik, Theor. Math. Phys. 61 (1984) 977 [Teor. Mat. Fiz. 61 (1984) 35].

[8] I. Cherednik, Int. J. Mod. Phys. A 7 (1992) 109.

[9] E. K. Sklyanin, J. Phys. A 21 (1988) 2375.

[10] P. P. Kulish and R. Sasaki, Prog. Theor. Phys. 89 (1993) 741 arXiv:hep-th/9212007.

[11] S. Ghoshal and A. B. Zamolodchikov, Int. J. Mod. Phys. A 9 (1994) 3841 [Erratum, ibid. A 9 (1994) 4353] arXiv:hep-th/9306002.

[12] E. Corrigan, P. E. Dorey and R. H. Rietdijk, Prog. Theor. Phys. Suppl. 118 (1995) 143 arXiv:hep-th/9407148.

[13] A. Fring and R. Koberle, Nucl. Phys. B $421 \quad$ (1994) 159 arXiv:hep-th/9304141.

[14] A. Liguori, M. Mintchev and L. Zhao, Commun. Math. Phys. 194, 569 (1998) arXiv:hep-th/9607085.

[15] G. Delfino, G. Mussardo and P. Simonetti, Nucl. Phys. B 432 (1994) 518 arXiv:hep-th/9409076. 
[16] R. Konik and A. LeClair, Nucl. Phys. B 538 (1999) 587 arXiv:hep-th/9703085.

[17] H. Saleur, Lectures on Non-perturbative field theory and quantum impurity problems, arXiv:cond-mat/9812110.

[18] H. Saleur, Lectures on Non-perturbative field theory and quantum impurity problems. II, arXiv:cond-mat/0007309.

[19] O. A. Castro-Alvaredo, A. Fring and F. Gohmann, On the absence of simultaneous reflection and transmission in integrable impurity systems, arXiv:hep-th/0201142.

[20] O. Castro-Alvaredo and A. Fring, Nucl. Phys. B 649 (2003) 449 arXiv:hep-th/0205076.

[21] M. Mintchev, E. Ragoucy and P. Sorba, Phys. Lett. B 547 (2002) 313 arXiv:hep-th/0209052.

[22] S. Albeverio, F. Gesztesy, R. Hoegh-Krohn ans H. Holden, Solvable models in quantum mechanics (Springer-Verlag, New York, 1988).

[23] M. Reed and B. Simon, Methods of modern mathematical physics III: Scattering theory (Academic Press, New York, 1979).

[24] M. Gattobigio, A. Liguori and M. Mintchev, J. Math. Phys. 40 (1999) 2949 arXiv:hep-th/9811188.

[25] A. Liguori, M. Mintchev and M. Rossi, J. Math. Phys. 38 (1997) 2888.

[26] M. Reed and B. Simon, Methods of modern mathematical physics II: Fourier analysis, self-adjointness (Academic Press, New York, 1975).

[27] A. Liguori and M. Mintchev, Commun. Math. Phys. 169 (1995) 635 arXiv:hep-th/9403039.

[28] A. Liguori and M. Mintchev, Lett. Math. Phys. 33 (1995) 283.

[29] M. Gattobigio, A. Liguori and M. Mintchev, Phys. Lett. B 428 (1998) 143 arXiv:hep-th/9801094.

[30] M. Mintchev, E. Ragoucy and P. Sorba, J. Phys. A 34 (2001) 8345 arXiv:hep-th/0104079. 
[31] E. Ragoucy, Lett. Math. Phys. 58 (2001) 249 arXiv:math.QA/0108221.

[32] C. Bachas, J. de Boer, R. Dijkgraaf and H. Ooguri, JHEP 0206 (2002) 027 arXiv:hep-th/0111210.

[33] O. DeWolfe, D. Z. Freedman and H. Ooguri, Phys. Rev. D 66 (2002) 025009 arXiv:hep-th/0111135. 